\title{
THE IRAS BRIGHT GALAXY SAMPLE. II. THE SAMPLE AND LUMINOSITY FUNCTION
}

\author{
B. T. Solfer,${ }^{1}$ D. B. SAnders, ${ }^{1}$ B. F. Madore, ${ }^{1,2,3}$ G. Neugebauer, ${ }^{1}$ G. E. Danielson, ${ }^{4}$ \\ J. H. Elias, ${ }^{1}$ Carol J. Lonsdale, ${ }^{5}$ AND W. L. RiCE ${ }^{5}$ \\ Received 1986 December 1; accepted 1987 February 13
}

\begin{abstract}
A complete sample of 324 extragalactic objects with $60 \mu \mathrm{m}$ flux densities greater than 5.4 Jy has been selected from the IRAS catalogs. Only one of these objects can be classified morphologically as a Seyfert nucleus; the others are all galaxies. The median distance of the galaxies in the sample is $\sim 30 \mathrm{Mpc}$, and the median luminosity $v L_{v}(60 \mu \mathrm{m})$ is $\sim 2 \times 10^{10} L_{\odot}$. This infrared selected sample is much more "infrared active" than optically selected galaxy samples.

The range in far-infrared luminosities of the galaxies in the sample is $10^{8} L_{\odot}-2 \times 10^{12} L_{\odot}$. The far-infrared luminosities of the sample galaxies appear to be independent of the optical luminosities, suggesting a separate luminosity component. As previously found, a correlation exists between $60 \mu \mathrm{m} / 100 \mu \mathrm{m}$ flux density ratio and far-infrared luminosity. The mass of interstellar dust required to produce the far-infrared radiation corresponds to a mass of gas of $10^{8}-10^{10} M_{\odot}$ for normal gas to dust ratios. This is comparable to the mass of the interstellar medium in most galaxies.

The infrared luminous galaxies are found to be an important component of extraglactic objects, being the most numerous objects in the local universe at luminosities $L>10^{11} L_{\odot}$, and producing a luminosity density of $\sim \frac{1}{4}$ that of the observed starlight in normal galaxies. Approximately $60 \%-80 \%$ of the far-infrared luminosity of the local universe is likely attributed to recent or ongoing star formation. If the infrared active phase $\left(L_{\text {FIR }}>10^{11} L_{\odot}\right)$ is a nonrecurring event of duration less than $10^{8} \mathrm{yr}$ in galaxy evolution, then more than $\sim 10 \%$, and perhaps all of the galaxies with blue luminosities greater than $10^{10} L_{\odot}$ must undergo such an event.
\end{abstract}

Subject headings: galaxies: general — infrared: general — infrared: sources — stars: formation

\section{INTRODUCTION}

The IRAS survey was the first infrared all-sky survey with sufficient sensitivity to detect a significant number of extragalactic sources. One result of the survey is the discovery that far-infrared emission dominates the total luminosity in a significant fraction of galaxies. Another, as discussed here, is the demonstration that the infrared luminous galaxies form a significant component of the local universe. To establish this, it is necessary to make a census of the galaxies that are infrared emitters, to determine the space densities of these galaxies, and to compare infrared bright galaxies with other known classes of extragalactic objects.

We have begun a study of the properties of the brightest infrared galaxies discovered in the IRAS survey with the goal of understanding the physical processes responsible for the infrared emission in galaxies. In this paper we describe a statistically complete sample of 324 objects selected for these studies, derive the far-infrared luminosity function of these galaxies, and compare the space densities of the IRAS bright galaxy sample with those of other major classes of extragalactic sources. A preliminary description of the results of the luminosity function for $L>10^{10} L_{\odot}$ was reported by Soifer $e t$ al. (1986, hereafter Paper I), and a detailed description of the

\footnotetext{
1 Palomar Observatory, California Institute of Technology.

2 Killam Fellow, Canada Council 1986-1988.

3 Also at the David Dunlap Observatory, University of Toronto.

4 Palomar Observatory and Division of Geology and Planetary Science, California Institute of Technology.

${ }^{5}$ IPAC, California Institute of Technology.
}

optical spectra and morphologies of the most luminous objects in this sample will be reported elsewhere (Sanders et al. 1987a).

\section{THE IRAS BRIGHT GALAXY SAMPLE}

The sample selected for study was designed to meet the following criteria: (1) it should be a complete sample of farinfrared-emitting extragalactic objects; (2) the size of the sample should be large enough to be able to make statistically significant statements regarding the space densities of infraredemitting objects; (3) the objects in the sample should be accessible from northern hemisphere telescopes; and (4) the optical identifications should be made with as little ambiguity as possible. The final bright galaxy sample comprised all extragalactic objects observed by IRAS with $60 \mu \mathrm{m}$ flux densities greater than $5.4 \mathrm{Jy}$, galactic latitude $|b|>30^{\circ}$, and declination $\delta>-30^{\circ}$ for $0-12 \mathrm{hr}, \delta>-15^{\circ}$ for $12-14 \mathrm{hr}$, and $\delta>-20^{\circ}$ for 14-24 hr. The declination boundaries indicate areas where redshift information is complete. An extension of the bright galaxy survey to the rest of the sky covered by $I R A S$ at $|b|>30^{\circ}$ and away from the Magallenic Clouds is currently under way (Elias, private communication).

For an object to be included in the IRAS bright galaxy sample it is necessary for it either to be identified with a cataloged extragalactic object or to have a redshift indicating it to be extragalactic. Although no morphological criteria were set for inclusion in the sample other than that there be an optical counterpart on the Palomar Sky Survey (POSS), all but one of the objects ultimately selected for inclusion in the sample are clearly extended on the POSS. The one exception to this is an 
object with a starlike Seyfert nucleus, IRAS 0518-25 (Sanders et al. $1987 a$ ).

The total area covered by the bright galaxy survey is $\sim 14,500 \mathrm{deg}^{2}$. Within the boundaries described above, small areas of the sky are not included because the.IRAS survey coverage was insufficient for the detected sources in this area to be included in the IRAS catalogs. The areas omitted are described in the IRAS Catalogs and Atlases Explanatory Supplement (1985). The major omission in areal coverage is a gap close to the North Galactic Pole. While this is a statistically insignificant gap in the all-sky survey and in the coverage of the IRAS bright galaxy sample, at least two objects, NGC 4258 and NGC 4151, were detected that would have qualified, with sufficient sky coverage, for inclusion in the bright galaxy sample.

Candidate sources were generated from three IRAS catalogs each sampling different spatial structures: the IRAS Point Source Catalog (1985, hereafter the PSC) for objects with sizes less than 2', the Small-Scale Structure Catalog (1986, hereafter the SSSC) for objects with sizes in the range $2^{\prime}-8^{\prime}$, and the Catalog of IRAS Observations of Large Galaxies (Rice et al. 1987, hereafter LGC) for larger objects. While there is no requirement that an object appear in the PSC, all objects that qualified for inclusion in the bright galaxy sample indeed have an entry in the PSC. Several of the brighter galaxies from the PSC are also listed in the SSSC or LGC. Of the total of 324 objects in the bright galaxy sample, 289 were selected from the PSC, eight were selected based on their $60 \mu \mathrm{m}$ flux density in the LGC, and 27 were selected from the SSSC.

Few of the objects selected from the PSC are Galactic sources. Of the 405 sources in the PSC meeting the areal and $60 \mu \mathrm{m}$ flux density constraints, 98 have $25 \mu \mathrm{m}$ flux densities greater than $60 \mu \mathrm{m}$ flux densities, and all of these are associated with Galactic sources. Of the remaining 307 objects, seven sources are associated with the local group galaxy M33, two with NGC 253, and 287 represent other individual galaxies. Of the remaining 11 sources, six are planetary nebulae, two are positionally coincident with bright SAO stars, one is a peculiar galactic object, IRAS $0937+12$, which is morphologically similar to the Egg Nebula (Ney et al. 1975; Kleinman, private communication), and two have no obvious optical counterparts. One of these latter two is in the direction of the dark nebula L1642 and can be identified with that source. The other, IRAS $1345+08$, has no obvious optical counterpart, although there is a very faint stellar object within $\sim 20^{\prime \prime}$ of the $I R A S$ position. No optical spectrum was obtained for this object, though it was assumed from its infrared energy distribution to be a Galactic source.

Of the objects included in the bright galaxy sample from the SSSC, all those with galaxy counterparts on the POSS have associations with previously cataloged bright galaxies. The candidate sources from the SSSC without counterparts in the PSC have neither associations with previously cataloged galaxies nor visible counterparts on the POSS, and are therefore attributed to high Galactic latitude "infrared cirrus" (Low et al. 1984). The existence of bright $60 \mu \mathrm{m}$ "cirrus" that produces such reproducible signatures has been confirmed by Low (private communication). There are no sources brighter than the 5.4 Jy limit of the bright galaxy sample and selected from the SSSC that can be identified as extragalactic and do not have comparatively bright counterparts on the POSS.

The LGC represents all galaxies known to have optical diameters greater than $8^{\prime}$. This catalog shows only one object out of $\sim 90$ (NGC 6822 ) that possibly has an optical diameter less than the infrared diameter, so that the inclusion of objects from this catalog probably implies that all objects meeting the areal constraints with infrared sizes greater than $8^{\prime}$ are included.

\section{THE DATA}

The observational data for the galaxies in the bright galaxy sample are given in Table 1. Except for the large galaxies, where the positions are taken from the Second Reference Catalogue of Bright Galaxies (de Vaucouleurs, de Vaucouleurs, and Corwin 1976), the positions for the galaxies are from the PSC.

The total $60 \mu \mathrm{m}$ and $100 \mu \mathrm{m}$ flux densities are taken (in decreasing order of priority) from the LGC, the SSSC, and the PSC. This order of selection of total flux density ensures that the estimate of the largest total flux density for a given galaxy has been used. Twenty-nine galaxies in the bright galaxy sample have $60 \mu \mathrm{m}$ flux densities taken from the LGC; 53 $60 \mu \mathrm{m}$ flux densities were obtained from the SSSC. In the case of NGC 5195, because of its proximity to M51, the $60 \mu \mathrm{m}$ and $100 \mu \mathrm{m}$ flux densities were estimated from one-dimensional co-addition of the IRAS survey data by subtracting a contribution from M51 that was assumed to be symmetrically distributed about the position of M51. Because the galaxies in this sample are all comparatively bright, the uncertainties in the reported flux densities are all dominated by systematic and calibration uncertainties, and should be less than $15 \%$.

The flux densities reported in Table 1 have been corrected for the large bandwidths of the IRAS $60 \mu \mathrm{m}$ and $100 \mu \mathrm{m}$ filters, assuming the intrinsic spectrum to be a Planck curve multiplied by an emissivity proportional to frequency. Typically the corrections are $30 \%-10 \%$ at $60 \mu \mathrm{m}$ and $<2 \%$ at $100 \mu \mathrm{m}$. The uncertainty in the intrinsic spectrum of the source leads to an uncertainty in the correction term of roughly $\pm 5 \%$ (see the IRAS Explanatory Supplement 1985).

Distances established from primary distance indicators (Sandage and Tammann 1981) or the Tully-Fisher relation (Aaronson et al. 1982a; Aaronson and Mould 1983) and modified for the distance adopted for the Virgo cluster (see below), have been adopted where available. For galaxies where neither of these are available, heliocentric radial velocities, from the literature (Palumbo, Tanzella-Nitta, and Vettolani, 1983; Huchra et al. 1983; Rood, private communication), were used in combination with a Hubble constant of $75 \mathrm{~km} \mathrm{~s}^{-1} \mathrm{Mpc}^{-1}$.

For those galaxies where no radial velocity was found in the literature, observations of the optical spectrum were made using the double spectrograph (Oke and Gunn 1982) on the $5 \mathrm{~m}$ Hale telescope of the Palomar Observatory. For these observations, the resolution was $12 \AA$ at $\mathrm{H} \alpha$, and the uncertainty in the radial velocity is $\pm 300 \mathrm{~km} \mathrm{~s}^{-1}$. In all cases where a galaxy redshift was determined from observations obtained at Palomar, the galaxy had strong emission lines of $\mathrm{H} \alpha$, [N II], [O III], and $\mathrm{H} \beta$. The optical spectra of these galaxies are discussed in detail by Sanders et al. (1987b).

Where distances were determined from redshifts, the distance to each galaxy was derived using the Virgocenric flow of Aaronson et al. (1982b). For all galaxies where distances were taken from the Fisher-Tully relation or the Virgocentric flow model the distances were scaled assuming Virgo is at $17.6 \mathrm{Mpc}$ and the infall velocity toward Virgo is $350 \mathrm{~km} \mathrm{~s}^{-1}$ (i.e., $H_{0}=$ $75 \mathrm{~km} \mathrm{~s}^{-1} \mathrm{Mpc}^{-1}$ at large distances). All galaxies within $6^{\circ}$ of the center of the Virgo cluster were assumed to be members of 
TABLE 1

IRAS BRIGHT GALAXY SAMPLE

\begin{tabular}{|c|c|c|c|c|c|c|c|c|c|c|c|c|c|}
\hline \multirow{3}{*}{$\begin{array}{l}\text { NAME } \\
\text { NGC } 23 .\end{array}$} & \multicolumn{3}{|c|}{ RA } & \multicolumn{3}{|c|}{ DEC } & \multicolumn{2}{|c|}{$\mathrm{F}_{u}$ (Jy) } & \multirow{3}{*}{$\begin{array}{c}\begin{array}{c}\mathrm{cz} \\
\mathrm{km} \mathrm{s}^{-1}\end{array} \\
4536\end{array}$} & \multirow{3}{*}{$\begin{array}{r}\mathrm{D} \\
\mathrm{Mpc}\end{array}$} & \multirow{3}{*}{$\begin{array}{c}\begin{array}{c}\log L_{\text {fir }} \\
\mathrm{L}_{\odot}\end{array} \\
10.93\end{array}$} & \multirow{3}{*}{$\begin{array}{c}\begin{array}{c}\mathrm{m}_{\mathrm{z}}^{2} \\
\mathrm{mag}\end{array} \\
12.5\end{array}$} & \multirow{3}{*}{$\begin{array}{c}\begin{array}{c}\text { Other } \\
\text { Name }\end{array} \\
\text { UGC } 89\end{array}$} \\
\hline & \multicolumn{6}{|c|}{$(1950)$} & $60 \mu \mathrm{m}$ & $100 \mu \mathrm{m}$ & & & & & \\
\hline & $0^{h}$ & $7^{\mathrm{m}}$ & $19: 4$ & $+25^{\circ}$ & $38^{\prime}$ & $46^{\prime \prime}$ & 9.6 & 16.0 & & & & & \\
\hline NGC 34 & 0 & 8 & 33.4 & -12 & 23 & 10 & 17.6 & 17.1 & 5931 & $\ldots$ & 11.34 & 13.0 & Mrk 938 \\
\hline MCG-02-01-051 & 0 & 16 & 18.0 & -10 & 39 & 14 & 7.1 & 9.1 & 7513 & $\cdots$ & 11.16 & $\ldots$ & Arp 256 \\
\hline NGC 150 & 0 & 31 & 47.3 & -28 & 4 & 44 & 10.1 & 17.5 & 1593 & $\ldots$ & 9.96 & 12.5 & \\
\hline NGC 157 & 0 & 32 & 13.9 & -8 & 40 & 23 & 18.6 & 37.8 & 1651 & $\ldots$ & 10.35 & 11.5 & \\
\hline NGC 174 & 0 & 34 & 31.4 & -29 & 45 & 11 & 12.1 & 19.2 & 3471 & $\ldots$ & 10.71 & 14.0 & \\
\hline NGC 232 & 0 & 40 & 17.5 & -23 & 50 & 2 & 10.7 & 18.7 & 6250 & $\ldots$ & 11.23 & 14.0 & \\
\hline NGC 247 & 0 & 44 & 40.0 & -21 & 2 & 0 & 10.5 & 30.2 & $\ldots$ & 3.6 & 8.67 & 9.8 & \\
\hline NGC 253 & 0 & 45 & 5.0 & -25 & 33 & 47 & 1245.0 & 2345.0 & $\ldots$ & 3.6 & 10.60 & 7.5 & \\
\hline UGC 556 & 0 & 52 & 7.7 & +28 & 58 & 26 & 6.1 & 9.9 & 4567 & $\ldots$ & 10.74 & 15.3 & \\
\hline NGC 337 & 0 & 57 & 19.9 & -7 & 50 & 53 & 8.6 & 17.2 & 1651 & $\ldots$ & 10.00 & 12.5 & \\
\hline IC 1623 & 1 & 5 & 18.0 & -17 & 46 & 37 & 22.6 & 28.9 & 5550 & $\ldots$ & 11.38 & 15.5 & VV114A/B \\
\hline MCG-03-04-014 & 1 & 7 & 42.0 & -17 & 7 & 1 & 7.7 & 9.9 & 10040 & $\ldots$ & 11.45 & 15.0 & \\
\hline NGC 470 & 1 & 17 & 9.6 & +3 & 8 & 53 & 6.7 & 12.0 & 2374 & $\ldots$ & 10.19 & 12.4 & UGC 858 \\
\hline $\mathrm{MCG}+02-04-025$ & 1 & 17 & 22.8 & +14 & 5 & 53 & 11.4 & 9.7 & 9337 & $\ldots$ & 11.55 & 14.5 & \\
\hline UGC 903 & 1 & 19 & 6.5 & +17 & 19 & 52 & 8.4 & 14.4 & 2320 & $\ldots$ & 10.29 & 14.7 & \\
\hline NGC 520 & 1 & 21 & 59.5 & +3 & 31 & 52 & 33.5 & 47.6 & 2261 & $\ldots$ & 10.79 & 12.4 & UGC 966 \\
\hline NGC 578 & 1 & 28 & 03.7 & -22 & 55 & 40 & 5.4 & 12.1 & 1696 & $\ldots$ & 9.81 & 11.5 & \\
\hline NGC 598 & 1 & 31 & 03.0 & +30 & 23 & 54 & 475.0 & 1724.0 & $\ldots$ & 0.8 & 9.11 & 6.5 & M33 \\
\hline NGC 613 & 1 & 31 & 59.0 & -29 & 40 & 34 & 24.2 & 49.1 & 1487 & $\ldots$ & 10.31 & 11.0 & \\
\hline NGC 628 & 1 & 34 & 1.0 & +15 & 31 & 36 & 22.8 & 65.2 & 655 & $\ldots$ & 9.87 & 10.5 & M74 \\
\hline IRAS 0136-10 & 1 & 36 & 24.0 & -10 & 42 & 25 & 7.0 & 6.2 & 14250 & $\ldots$ & 11.71 & $\ldots$ & \\
\hline NGC 660 & 1 & 40 & 21.6 & +13 & 23 & 42 & 76.1 & 107.1 & 862 & $\ldots$ & 10.38 & 12.8 & UGC 1201 \\
\hline III Zw 035 & 1 & 41 & 48.0 & +16 & 51 & 7 & 13.8 & 13.3 & 8215 & $\ldots$ & 11.54 & $15.8^{1}$ & \\
\hline NGC 693 & 1 & 47 & 54.2 & +5 & 53 & 53 & 7.9 & 11.0 & 1593 & $\ldots$ & 9.86 & 13.5 & UGC 1304 \\
\hline NGC 695 & 1 & 48 & 28.1 & +22 & 20 & 10 & 8.6 & 13.2 & 9769 & $\ldots$ & 11.52 & 13.5 & UGC 1315 \\
\hline NGC 701 & 1 & 48 & 35.0 & -9 & 57 & 0 & 6.5 & 13.6 & $\ldots$ & 18.9 & 9.80 & 13.0 & \\
\hline UGC 1351 & 1 & 50 & 18.7 & +12 & 27 & 43 & 6.6 & 12.3 & 4597 & $\ldots$ & 10.78 & 14.0 & IC 1743 \\
\hline UGC 1451 & 1 & 55 & 41.5 & +25 & 7 & 5 & 7.1 & 12.9 & 4916 & $\ldots$ & 10.88 & 14.3 & \\
\hline NGC 772 & 1 & 56 & 34.6 & +18 & 45 & 52 & 8.1 & 21.0 & $\ldots$ & 33.1 & 10.47 & 11.3 & Arp 78 \\
\hline NGC 835 & 2 & 6 & 56.6 & -10 & 22 & 23 & 6.2 & 10.7 & 4066 & $\ldots$ & 10.60 & 13.5 & Arp 318 \\
\hline UGC 1720 & 2 & 11 & 28.3 & +4 & 56 & 28 & 5.6 & 8.2 & 3448 & $\ldots$ & 10.39 & 14.4 & Mrk 1027 \\
\hline NGC 873 & 2 & 14 & 5.3 & -11 & 34 & 55 & 6.2 & 12.1 & 3450 & $\ldots$ & 10.47 & 13.0 & \\
\hline NGC 877 & 2 & 15 & 15.1 & +14 & 18 & 36 & 12.4 & 24.3 & 3866 & $\ldots$ & 10.91 & 12.5 & UGC 1768 \\
\hline NGC 908 & 2 & 20 & 46.6 & -21 & 27 & 36 & 14.4 & 44.7 & 1508 & $\ldots$ & 10.23 & 11.0 & \\
\hline NGC 922 & 2 & 22 & 49.4 & -25 & 0 & 54 & 5.5 & 9.5 & 3086 & $\ldots$ & 10.28 & 12.5 & \\
\hline NGC 958 & 2 & 28 & 11.8 & -3 & 9 & 32 & 5.5 & 15.2 & 5750 & $\cdots$ & 11.02 & 11.5 & \\
\hline NGC 992 & 2 & 34 & 35.8 & +20 & 53 & 6 & 10.7 & 16.6 & 4119 & $\ldots$ & 10.85 & 13.5 & UGC 2103 \\
\hline NGC 1022 & 2 & 36 & 4.6 & -6 & 53 & 31 & 21.0 & 26.7 & 1498 & $\ldots$ & 10.18 & $\ldots$ & \\
\hline NGC 1055 & 2 & 39 & 11.8 & +0 & 13 & 52 & 21.7 & 60.8 & 1005 & $\ldots$ & 10.08 & 12.5 & UGC 2173 \\
\hline NGC 1068 & 2 & 40 & 7.2 & -0 & 13 & 30 & 188.9 & 238.7 & 1125 & $\ldots$ & 10.90 & 9.7 & M77 \\
\hline NGC 1083 & 2 & 43 & 18.7 & -15 & 34 & 5 & 7.7 & 14.1 & 5040 & $\ldots$ & 10.89 & 14.0 & \\
\hline NGC 1084 & 2 & 43 & 32.4 & -7 & 47 & 13 & 27.4 & 55.1 & 1402 & $\ldots$ & 10.34 & 12.0 & \\
\hline UGC 2238 & 2 & 43 & 33.4 & +12 & 53 & 10 & 8.8 & 15.7 & 6250 & $\ldots$ & 11.16 & 15.2 & \\
\hline IRAS $0243+21$ & 2 & 43 & 49.2 & +21 & 22 & 44 & 6.2 & 6.5 & 6810 & $\ldots$ & 11.00 & & \\
\hline NGC 1087 & 2 & 43 & 51.8 & -0 & 42 & 25 & 9.6 & 29.6 & 1503 & $\ldots$ & 10.10 & 11.5 & UGC 2245 \\
\hline NGC 1134 & 2 & 50 & 57.1 & +12 & 48 & 43 & 9.6 & 17.5 & 3595 & $\ldots$ & 10.72 & 13.2 & UGC 2365 \\
\hline UGC 2369 & 2 & 51 & 15.6 & +14 & 46 & 1 & 8.0 & 11.9 & 9354 & $\ldots$ & 11.44 & 14.6 & \\
\hline NGC $1143 / 4$ & 2 & 52 & 38.6 & -0 & 23 & 6 & 5.6 & 11.4 & 8550 & $\ldots$ & 11.28 & 13.2 & UGC $2388 / 9$ \\
\hline UGC 2403 & 2 & 53 & 23.0 & +0 & 29 & 28 & 7.8 & 11.3 & 5450 & $\ldots$ & 10.93 & 14.9 & \\
\hline NGC 1187 & 3 & 0 & 23.8 & -23 & 3 & 43 & 10.3 & 23.4 & 1394 & $\cdots$ & 9.90 & 11.0 & \\
\hline
\end{tabular}

the cluster, and their distances are taken as $17.6 \mathrm{Mpc}$. In addition the galaxies within $20^{\circ}$ of the center of Virgo, whose distances are not derived from another source, and whose radial velocity is within $400 \mathrm{~km} \mathrm{~s}^{-1}$ of that of Virgo, are assumed to be cluster members. All distance and redshift information used for the bright galaxy sample is given in Table 1 .

\section{COMPLETENESS OF THE SAMPLE}

Because the completeness limit of the PSC at $60 \mu \mathrm{m}$ is $\sim 0.5 \mathrm{Jy}$ (IRAS Explanatory Supplement 1985) the IRAS bright galaxy sample should be highly complete. The completeness of the PSC is well understood (Chester 1986; IRAS
Explanatory Supplement 1985). The SSSC is estimated to be complete above $10 \mathrm{Jy}$ at $60 \mu \mathrm{m}$, although this has not been investigated in detail (SSSC Introduction, 1986). Thus there could be some incompleteness in sources selected from the SSSC for inclusion in the bright galaxy sample. This is unlikely to be significant since the differential number counts with the flux density of the sources in the bright galaxy sample in Figure 1 shows an acceptable fit to an $N \approx f_{v}{ }^{-3 / 2}$ distribution to the lowest flux bin. It therefore appears that the bright galaxy sample is, to a good approximation, a complete and unbiased sample of $60 \mu \mathrm{m}$ extragalactic sources. Note that the objects in the bright galaxy sample have been selected on the basis of 
TABLE 1-Continued

\begin{tabular}{|c|c|c|c|c|c|c|c|c|c|c|c|c|c|}
\hline \multirow{3}{*}{$\begin{array}{l}\text { NAME } \\
\text { NGC } 1204\end{array}$} & \multicolumn{3}{|c|}{ RA } & \multicolumn{3}{|c|}{ DEC } & \multicolumn{2}{|c|}{$\mathrm{F}_{\nu},(\mathrm{Jy})$} & \multirow{3}{*}{$\begin{array}{c}\begin{array}{c}\mathrm{cz} \\
\mathrm{km} \mathrm{s}^{-1}\end{array} \\
4282\end{array}$} & \multirow{3}{*}{$\begin{array}{c}\mathrm{D} \\
\mathrm{Mpc}\end{array}$} & \multirow{3}{*}{$\frac{\begin{array}{c}\log L_{\text {air }} \\
L_{C}\end{array}}{10.70}$} & \multirow{3}{*}{$\frac{\begin{array}{c}\mathrm{m}_{\mathrm{z}}^{2} \\
\mathrm{mag}\end{array}}{15.0}$} & \multirow{2}{*}{$\begin{array}{l}\text { Other } \\
\text { Name }\end{array}$} \\
\hline & \multicolumn{6}{|c|}{$(1950)$} & $60 \mu \mathrm{m}$ & $100 \mu \mathrm{m}$ & & & & & \\
\hline & 3 & 2 & 16.8 & -12 & 32 & 6 & 8.1 & 10.4 & & & & & \\
\hline NGC 1222 & 3 & 6 & 24.2 & -3 & 8 & 49 & 13.2 & 15.3 & 2600 & $\ldots$ & 10.46 & $\ldots$ & \\
\hline NGC 1232 & 3 & 7 & 28.3 & -20 & 45 & 49 & 10.9 & 40.9 & 1720 & $\cdots$ & 10.31 & 11.1 & Arp 41 \\
\hline NGC 1266 & 3 & 13 & 28.6 & -2 & 36 & 43 & 11.7 & 16.6 & 2035 & $\ldots$ & 10.22 & 14.0 & \\
\hline NGC 1309 & 3 & 19 & 46.1 & -15 & 34 & 34 & 5.7 & 14.0 & 2138 & $\ldots$ & 10.06 & 12.5 & \\
\hline IC 1953 & 3 & 31 & 29.5 & -21 & 38 & 42 & 9.1 & 11.1 & 1995 & $\ldots$ & 10.03 & 12.5 & \\
\hline NGC 1377 & 3 & 34 & 25.7 & -21 & 3 & 58 & 7.1 & 5.5 & 1474 & $\ldots$ & 9.66 & 14.0 & \\
\hline NGC 1385 & 3 & 35 & 19.7 & -24 & 39 & 47 & 16.8 & 35.4 & 1488 & $\ldots$ & 10.15 & 12.0 & \\
\hline IRAS $0335+15$ & 3 & 35 & 57.1 & +15 & 23 & 6 & 5.9 & 7.0 & 10600 & $\ldots$ & 11.38 & $\ldots$ & \\
\hline NGC 1415 & 3 & 38 & 45.6 & -22 & 43 & 30 & 5.6 & 12.1 & 1617 & $\cdots$ & 9.76 & 12.5 & \\
\hline NGC 1421 & 3 & 40 & 8.9 & -13 & 38 & 49 & 12.1 & 21.7 & 2099 & $\ldots$ & 10.29 & 12.0 & \\
\hline NGC 1482 & 3 & 52 & 25.9 & -20 & 38 & 53 & 33.1 & 45.6 & 1655 & $\ldots$ & 10.44 & 14.0 & \\
\hline UGC 2982 & 4 & 9 & 43.2 & +5 & 25 & 12 & 8.9 & 16.0 & 5321 & $\cdots$ & 11.02 & 15.5 & \\
\hline MCG-03-12-002 & 4 & 19 & 6.5 & -18 & 55 & 48 & 5.8 & 9.1 & 9477 & $\ldots$ & 11.30 & 15.5 & \\
\hline NGC 1614 & 4 & 31 & 35.8 & -8 & 40 & 55 & 34.0 & 31.1 & 4745 & $\ldots$ & 11.41 & 15.0 & Mrk 617 \\
\hline IRAS $0433-25$ & 4 & 33 & 35.0 & -25 & 14 & 6 & 5.6 & 9.6 & 4843 & $\cdots$ & 10.70 & $\ldots$ & \\
\hline MCG-04-12-003 & 4 & 37 & 1.0 & -24 & 16 & 52 & 6.3 & 11.2 & 4422 & $\ldots$ & 10.68 & 14.0 & \\
\hline NGC 1637 & 4 & 38 & 57.1 & -2 & 57 & 11 & 5.9 & 13.5 & 726 & $\ldots$ & 9.13 & 11.5 & \\
\hline NGC 1667 & 4 & 46 & 9.8 & -6 & 24 & 29 & 6.1 & 14.5 & 4600 & $\ldots$ & 10.80 & $\ldots$ & \\
\hline IRAS $0518-25$ & 5 & 18 & 58.6 & -25 & 24 & 40 & 13.8 & 11.0 & 12706 & $\ldots$ & 11.89 & $15.4^{1}$ & \\
\hline IRAS $0833+65$ & 8 & 33 & 55.4 & +65 & 17 & 49 & 6.2 & 6.5 & 5608 & $\ldots$ & 10.88 & $\ldots$ & \\
\hline NGC 2623 & 8 & 35 & 25.2 & +25 & 55 & 48 & 25.6 & 27.3 & 5538 & $\ldots$ & 11.47 & 14.5 & Arp 243 \\
\hline NGC 2633 & 8 & 42 & 32.9 & +74 & 16 & 59 & 16.9 & 26.5 & 2157 & $\ldots$ & 10.65 & 12.5 & UGC 4574 \\
\hline NGC 2683 & 8 & 49 & 35.0 & +33 & 36 & 30 & 10.3 & 34.5 & 284 & $\ldots$ & 8.76 & 9.7 & \\
\hline NGC 2681 & 8 & 50 & 0.7 & +51 & 30 & 4 & 7.5 & 11.0 & 720 & $\ldots$ & 9.48 & 10.4 & UGC 4645 \\
\hline IRAS $0857+39$ & 8 & 57 & 13.0 & +39 & 15 & 40 & 7.2 & 4.2 & 17480 & $\cdots$ & 11.99 & $15.0^{1}$ & \\
\hline NGC 2748 & 9 & 8 & 1.0 & +76 & 40 & 52 & 7.2 & 19.3 & 1489 & $\ldots$ & 10.20 & 11.7 & UGC 4825 \\
\hline NGC 2782 & 9 & 10 & 54.0 & +40 & 19 & 12 & 8.8 & 13.4 & 2552 & $\ldots$ & 10.46 & 12.3 & Arp 215 \\
\hline NGC 2785 & 9 & 12 & 2.9 & +41 & 7 & 34 & 9.2 & 16.3 & 2737 & $\cdots$ & 10.57 & 14.9 & UGC 4876 \\
\hline UGC 4881 & 9 & 12 & 39.6 & +44 & 32 & 20 & 6.3 & 9.9 & 11957 & $\ldots$ & 11.59 & 14.9 & Arp 55 \\
\hline NGC 2798 & 9 & 14 & 11.0 & +42 & 12 & 29 & 23.8 & 28.4 & 1755 & $\cdots$ & 10.59 & 12.9 & UGC 4905 \\
\hline NGC 2820 & 9 & 17 & 43.2 & +64 & 28 & 14 & 5.5 & 9.5 & 1686 & $\ldots$ & 10.02 & 13.1 & UGC 4961 \\
\hline NGC 2856 & 9 & 20 & 53.3 & +49 & 27 & 50 & 5.9 & 8.8 & 2638 & $\ldots$ & 10.32 & 13.9 & Arp 285 \\
\hline NGC 2903 & 9 & 29 & 19.9 & +21 & 43 & 23 & 59.5 & 154.9 & 554 & $\ldots$ & 9.93 & 9.8 & UGC 5079 \\
\hline UGC 5101 & 9 & 32 & 4.6 & +61 & 34 & 37 & 12.8 & 19.6 & 12000 & $\ldots$ & 11.90 & 15.5 & \\
\hline MCG+08-18-012 & 9 & 33 & 18.5 & +48 & 41 & 53 & 6.2 & 8.1 & 7790 & $\ldots$ & 11.19 & 15.0 & \\
\hline NGC 2967 & 9 & 39 & 29.3 & +0 & 33 & 58 & 5.4 & 15.0 & 1887 & $\ldots$ & 10.17 & 12.2 & UGC 5180 \\
\hline NGC 2966 & 9 & 39 & 34.1 & +4 & 54 & 7 & 5.7 & 8.0 & 2048 & $\cdots$ & 10.06 & 14.0 & UGC 5181 \\
\hline NGC 2964 & 9 & 39 & 55.7 & +32 & 4 & 37 & 12.4 & 23.7 & 1319 & $\ldots$ & 10.20 & 12.0 & Mrk 404 \\
\hline NGC 2976 & 9 & 43 & 6.2 & +68 & 9 & 22 & 10.7 & 29.6 & $\ldots$ & 3.4 & 8.62 & 10.9 & UGC 5221 \\
\hline NGC 2990 & 9 & 43 & 40.6 & +5 & 56 & 20 & 5.4 & 9.4 & 3155 & $\ldots$ & 10.42 & 12.5 & UGC 5229 \\
\hline IC $563 / 4$ & 9 & 43 & 44.2 & +3 & 17 & 26 & 6.6 & 10.4 & 6100 & $\ldots$ & 11.01 & 14.1 & Arp 303 \\
\hline NGC 2985 & 9 & 45 & 54.0 & +72 & 30 & 43 & 5.8 & 19.4 & 1277 & $\ldots$ & 10.10 & 11.1 & UGC 5253 \\
\hline NGC 3044 & 9 & 51 & 6.2 & +1 & 48 & 54 & 9.8 & 20.0 & 1318 & $\ldots$ & 10.06 & 12.4 & UGC 5311 \\
\hline NGC 3031 & 9 & 51 & 29.0 & +69 & 18 & 4 & 49.0 & 177.7 & $\ldots$ & 3.4 & 9.39 & 8.1 & M81 \\
\hline NGC 3034 & 9 & 51 & 42.5 & +69 & 54 & 58 & 1198.4 & 1129.9 & $\ldots$ & 3.4 & 10.45 & 9.2 & M82 \\
\hline NGC 3067 & 9 & 55 & 26.2 & +32 & 36 & 32 & 9.6 & 18.9 & 1506 & $\ldots$ & 10.20 & 12.7 & UGC 5351 \\
\hline NGC 3079 & 9 & 58 & 35.0 & +55 & 55 & 16 & 45.9 & 89.4 & $\ldots$ & 19.2 & 10.63 & 11.2 & UGC 5387 \\
\hline NGC 3094 & 9 & 58 & 42.0 & +16 & 0 & 43 & 11.3 & 13.8 & 2388 & $\ldots$ & 10.48 & 13.5 & UGC 5390 \\
\hline NGC 3077 & 9 & 59 & 17.0 & +68 & 58 & 37 & 15.6 & 24.6 & $\ldots$ & 3.4 & 8.60 & 10.7 & UGC 5398 \\
\hline NGC 3110 & 10 & 1 & 32.2 & -6 & 14 & 2 & 11.6 & 21.5 & 4840 & $\ldots$ & 11.10 & 13.5 & \\
\hline
\end{tabular}

$60 \mu \mathrm{m}$ flux density, not total far-infrared or total infrared flux, and all statements regarding completeness apply only at $60 \mu \mathrm{m}$.

\section{BASIC PROPERTIES OF THE BRIGHT GALAXY SAMPLE}

Figure 2 shows the distribution of distances to the galaxies in the bright galaxy sample. These galaxies range in distances from $0.6 \mathrm{Mpc}$ for M33 to greater than $300 \mathrm{Mpc}$ for the most distant galaxies in the sample. The median distance for the galaxies in the sample, excluding Virgo galaxies, is $32 \mathrm{Mpc}$. Thus the IRAS bright galaxy sample extends well beyond the Local Supercluster, but is not sampling objects at distances significant with respect to the size of the universe. A total of 31 sample galaxies are identified as associated with the Virgo cluster. Thus the Virgo cluster presents a $10 \%$ contribution to the bright galaxy sample.

Histograms of the far-infrared and blue luminosities of the objects in the bright galaxy sample are plotted in Figure 3. The total far-infrared luminosity $L_{\mathrm{FIR}}$ for the galaxy is calculated using the far-infrared flux, $f_{\text {FIR }}$, which is derived by fitting the $60 \mu \mathrm{m}$ and $100 \mu \mathrm{m}$ flux densities to a single temperature Planck function multiplied by an emissivity $\epsilon \propto v$ (Cataloged Galaxies and Quasars Observed in the IRAS Survey, Appendix B, 1985). For luminosity calculations the deceleration constant $q_{0}$ was assumed to be zero. The blue luminosity is the quantity $v L_{v}(0.43 \mu \mathrm{m})$ and is derived from the Zwicky (blue) magnitudes given in Table 1 . The blue flux, $f_{b} \equiv v f_{v}(0.43 \mu \mathrm{m})$, has been estimated from the relation between $m_{b}$ and $m_{z}$ suggested by 
TABLE 1-Continued

\begin{tabular}{|c|c|c|c|c|c|c|c|c|c|c|c|c|c|}
\hline \multirow{3}{*}{$\begin{array}{l}\text { NAME } \\
\text { NGC } 3166\end{array}$} & \multicolumn{3}{|c|}{ RA } & \multicolumn{3}{|c|}{ DEC } & \multicolumn{2}{|c|}{$\mathrm{F}_{\nu}(\mathrm{Jy})$} & \multirow{3}{*}{$\frac{\mathrm{km} \mathrm{s}^{-1}}{1381}$} & \multirow{3}{*}{$\begin{array}{c}\mathrm{D} \\
\mathrm{Mpc}\end{array}$} & \multirow{3}{*}{$\begin{array}{c}\begin{array}{c}\log L_{\text {fir }} \\
L_{\odot}\end{array} \\
9.93\end{array}$} & \multirow{3}{*}{$\begin{array}{r}\begin{array}{r}\mathrm{m}_{\mathrm{z}}{ }^{\mathrm{a}} \\
\mathrm{mag}\end{array} \\
11.2\end{array}$} & \multirow{3}{*}{$\begin{array}{c}\begin{array}{c}\text { Other } \\
\text { Name }\end{array} \\
\text { UGC } 5516\end{array}$} \\
\hline & \multicolumn{6}{|c|}{$(1950)$} & $60 \mu \mathrm{m}$ & $100 \mu \mathrm{m}$ & & & & & \\
\hline & 10 & 11 & 11.8 & +3 & 40 & 12 & 5.9 & 13.3 & & & & & \\
\hline NGC 3169 & 10 & 11 & 39.6 & +3 & 42 & 50 & 7.0 & 19.8 & 1205 & $\ldots$ & 9.98 & 11.9 & UGC 5525 \\
\hline NGC 3147 & 10 & 12 & 38.4 & +73 & 39 & 0 & 6.9 & 24.7 & 2820 & $\ldots$ & 10.74 & 11.3 & UGC 5532 \\
\hline NGC 3177 & 10 & 13 & 48.5 & +21 & 22 & 23 & 9.6 & 17.8 & 1303 & $\ldots$ & 10.10 & 12.8 & UGC 5544 \\
\hline NGC 3184 & 10 & 15 & 16.4 & +41 & 40 & 28 & 7.8 & 28.0 & 589 & $\ldots$ & 9.43 & 10.3 & UGC 5557 \\
\hline NGC 3198 & 10 & 16 & 52.0 & +45 & 48 & 00 & 7.4 & 19.2 & 665 & $\ldots$ & 9.52 & 10.7 & \\
\hline IRAS $1017+08$ & 10 & 17 & 22.1 & +8 & 28 & 41 & 6.1 & 5.4 & 14390 & $\ldots$ & 11.68 & $\ldots$ & \\
\hline NGC 3221 & 10 & 19 & 33.4 & +21 & 49 & 34 & 7.3 & 18.7 & 3971 & $\ldots$ & 10.89 & 14.3 & UGC 5601 \\
\hline NGC 3227 & 10 & 20 & 46.6 & +20 & 7 & 8 & 8.2 & 17.3 & 1110 & $\ldots$ & 9.95 & 12.0 & UGC 5620 \\
\hline NGC 3294 & 10 & 33 & 23.5 & +37 & 34 & 59 & 6.3 & 17.1 & 1592 & $\ldots$ & 10.21 & 11.5 & UGC 5753 \\
\hline NGC 3310 & 10 & 35 & 39.6 & +53 & 45 & 50 & 34.8 & 40.7 & 970 & $\ldots$ & 10.39 & 11.0 & Arp 217 \\
\hline NGC 3344 & 10 & 40 & 46.4 & +25 & 11 & 07 & 8.9 & 20.1 & 585 & $\ldots$ & 8.98 & 11.8 & \\
\hline NGC 3351 & 10 & 41 & 19.0 & +11 & 58 & 1 & 18.3 & 35.1 & 776 & $\ldots$ & 9.42 & 10.7 & M95 \\
\hline NGC 3353 & 10 & 42 & 15.1 & +56 & 13 & 30 & 5.4 & 6.4 & 940 & $\ldots$ & 9.57 & 12.9 & Mrk 35 \\
\hline NGC 3359 & 10 & 43 & 21.1 & +63 & 29 & 04 & 7.1 & 13.8 & $\ldots$ & 19.4 & 9.83 & 11.0 & UGC 5873 \\
\hline NGC 3367 & 10 & 43 & 54.7 & +14 & 0 & 58 & 6.1 & 12.8 & 3040 & $\ldots$ & 10.53 & 12.0 & UGC 5880 \\
\hline NGC 3368 & 10 & 44 & 7.7 & +12 & 4 & 59 & 9.6 & 27.4 & 899 & $\ldots$ & 9.45 & 12.0 & M96 \\
\hline NGC $3395 / 6$ & 10 & 47 & 4.3 & +33 & 15 & 0 & 11.6 & 16.6 & 1630 & $\ldots$ & 10.31 & 12.1 & Arp 270 \\
\hline NGC 3424 & 10 & 48 & 59.8 & +33 & 9 & 54 & 9.5 & 17.1 & 1494 & $\ldots$ & 10.22 & 13.2 & UGC 5972 \\
\hline NGC 3432 & 10 & 49 & 44.2 & +36 & 53 & 26 & 8.0 & 12.4 & $\ldots$ & 10.9 & 9.32 & 11.6 & Arp 206 \\
\hline NGC 3437 & 10 & 49 & 52.8 & +23 & 12 & 4 & 12.2 & 20.3 & 1291 & $\ldots$ & 10.23 & 12.6 & UGC 5995 \\
\hline NGC 3448 & 10 & 51 & 38.4 & +54 & 34 & 19 & 5.9 & 10.9 & 1380 & $\ldots$ & 9.96 & 12.2 & Arp 205 \\
\hline NGC 3471 & 10 & 56 & 2.2 & +61 & 47 & 53 & 8.9 & 11.8 & 2254 & $\ldots$ & 10.39 & 13.0 & Mrk 158 \\
\hline IRAS $1056+24$ & 10 & 56 & 35.5 & +24 & 48 & 43 & 12.7 & 13.8 & 12501 & $\ldots$ & 11.87 & $\ldots$ & \\
\hline NGC 3486 & 10 & 57 & 40.0 & +29 & 14 & 44 & 7.0 & 13.5 & 720 & $\ldots$ & 9.06 & 11.1 & UGC 6079 \\
\hline NGC 3504 & 11 & 0 & 28.6 & +28 & 14 & 28 & 20.0 & 32.4 & 1549 & $\ldots$ & 10.55 & 11.5 & UGC 6118 \\
\hline NGC 3508 & 11 & 0 & 30.7 & -16 & 1 & 12 & 7.5 & 13.9 & 3580 & $\ldots$ & 10.66 & 14.0 & \\
\hline NGC 3511 & 11 & 0 & 56.2 & -22 & 48 & 58 & 9.3 & 21.1 & 1226 & $\ldots$ & 9.94 & 11.5 & \\
\hline A1101+41 & 11 & 1 & 5.8 & +41 & 7 & 8 & 6.5 & 10.4 & 10350 & $\ldots$ & 11.50 & 15.0 & V32 \\
\hline NGC 3521 & 11 & 3 & 14.2 & +0 & 14 & 6 & 50.0 & 130.5 & 804 & $\cdots$ & 9.94 & 10.1 & UGC 6150 \\
\hline NGC 3556 & 11 & 8 & 35.3 & +55 & 56 & 46 & 35.3 & 83.8 & 697 & $\ldots$ & 10.30 & 10.9 & M108 \\
\hline NGC 3583 & 11 & 11 & 23.0 & +48 & 35 & 17 & 7.3 & 17.7 & 2135 & $\ldots$ & 10.42 & 11.6 & UGC 6263 \\
\hline NGC 3593 & 11 & 11 & 59.8 & +13 & 5 & 28 & 20.4 & 35.5 & 621 & $\ldots$ & 9.16 & 11.8 & UGC 6272 \\
\hline NGC 3597 & 11 & 12 & 14.4 & -23 & 27 & 18 & 13.8 & 16.8 & 3300 & $\ldots$ & 10.77 & 13.0 & \\
\hline NGC 3627 & 11 & 17 & 39.6 & +13 & 15 & 36 & 62.5 & 151.3 & $\ldots$ & 9.9 & 10.25 & 8.9 & M66 \\
\hline NGC 3628 & 11 & 17 & 41.8 & +13 & 51 & 40 & 54.0 & 127.8 & $\ldots$ & 9.9 & 10.18 & 11.5 & UGC 6350 \\
\hline NGC 3631 & 11 & 18 & 12.0 & +53 & 26 & 38 & 12.0 & 25.0 & 1161 & $\ldots$ & 10.21 & 11.0 & Arp 27 \\
\hline $\mathrm{MCG}+00-29-023$ & 11 & 18 & 38.6 & -2 & 42 & 36 & 5.7 & 8.9 & 7230 & $\ldots$ & 11.10 & 15.0 & \\
\hline NGC 3655 & 11 & 20 & 17.5 & +16 & 51 & 50 & 7.9 & 18.9 & 1481 & $\ldots$ & 9.85 & 11.6 & UGC 6396 \\
\hline NGC 3672 & 11 & 22 & 30.0 & -9 & 31 & 12 & 9.3 & 22.7 & 1855 & $\cdots$ & 10.37 & 12.0 & \\
\hline UGC 6436 & 11 & 23 & 9.8 & +14 & 56 & 53 & 6.9 & 10.0 & 10216 & $\ldots$ & 11.48 & 15.4 & IC 2810 \\
\hline NGC 3675 & 11 & 23 & 25.4 & +43 & 51 & 32 & 12.5 & 35.6 & 771 & $\cdots$ & 9.86 & 10.4 & UGC 6439 \\
\hline NGC 3683 & 11 & 24 & 42.7 & +57 & 9 & 7 & 14.7 & 29.3 & 1656 & $\ldots$ & 10.49 & 12.7 & UGC 6458 \\
\hline NGC 3690 & 11 & 25 & 42.0 & +58 & 50 & 17 & 108.9 & 108.6 & 3159 & $\ldots$ & 11.72 & 11.8 & Mrk 171 \\
\hline NGC 3726 & 11 & 30 & 37.3 & +47 & 18 & 16 & 6.7 & 21.3 & $\ldots$ & 16.7 & 9.87 & 11.1 & UGC 6537 \\
\hline NGC 3735 & 11 & 33 & 0.5 & +70 & 48 & 50 & 7.5 & 17.8 & 2696 & $\ldots$ & 10.58 & 12.6 & UGC 6567 \\
\hline NGC 3810 & 11 & 38 & 23.8 & +11 & 44 & 53 & 13.0 & 31.4 & $\ldots$ & 17.7 & 10.07 & 11.4 & UGC 6644 \\
\hline NGC 3877 & 11 & 43 & 29.5 & +47 & 46 & 16 & 8.3 & 18.9 & $\ldots$ & 17.4 & 9.85 & 11.8 & UGC 6745 \\
\hline NGC 3885 & 11 & 44 & 16.6 & -27 & 38 & 53 & 11.9 & 14.8 & 1948 & $\ldots$ & 10.30 & 13.0 & \\
\hline
\end{tabular}

Kirschner, Oemler, and Schechter (1978) and the absolute calibration of the blue magnitude given by Allen (1973). In all plots that follow where a blue flux is required, this conversion to the $m_{b}$ system has been applied. Since one of the selection criteria for the bright galaxy sample was high galactic latitude, extinction in the blue has been neglected, as have corrections for inclination or internal reddening.

The range of observed far-infrared luminosities extends from $\sim 10^{8} L_{\odot}$ to greater than $10^{12} L_{\odot}$, with the mode of the distribution occurring at $\sim 2 \times 10^{10} L_{\odot}$. A similar distribution (adjusted to the same Hubble constant) was found by Lawrence et al. 1986. All the sources in the bright galaxy sample have far-infrared flux densities much greater than can be attributed directly to a stellar population, while none are known radio-loud objects where the infrared emission could be expected to be an extension of the radio nonthermal emission. Furthermore, many of the objects in the sample show spatial extent at $60 \mu \mathrm{m}$. Thus, we assume that the far-infrared peak in the energy distribution is due to thermal emission by dust. For far-infrared luminosities of $\sim 2 \times 10^{10} L_{\odot}$ and typical dust temperatures of $\sim 35 \mathrm{~K}$, the mass of dust required to produce the observed luminosity is $\sim 4 \times 10^{6} M_{\odot}$ assuming optically thin dust emission and normal dust parameters (e.g., Draine and Lee 1984). This corresponds to a total gas mass of $\sim 10^{9}$ $M_{\odot}$, quite typical for the interstellar medium of large spiral galaxies.

As can be seen from Figure 3 the mean blue luminosity is significantly lower than the far-infrared luminosity, while the dispersion in the blue luminosities is about half that in the far-infrared luminosities. For the 312 galaxies with blue 
TABLE 1-Continued

\begin{tabular}{|c|c|c|c|c|c|c|c|c|c|c|c|c|c|}
\hline \multirow{3}{*}{$\begin{array}{l}\text { NAME } \\
\text { NGC } 3887\end{array}$} & \multicolumn{3}{|c|}{ RA } & \multicolumn{3}{|c|}{ DEC } & \multicolumn{2}{|c|}{$\mathrm{F}_{\nu}(\mathrm{Jy})$} & \multirow{3}{*}{$\frac{\begin{array}{c}\mathrm{cz} \\
\mathrm{km} \mathrm{s}^{-1}\end{array}}{1130}$} & \multirow{3}{*}{$\begin{array}{c}\mathrm{D} \\
\mathrm{Mpc}\end{array}$} & \multirow{3}{*}{$\begin{array}{c}\begin{array}{c}\log L_{\text {fir }} \\
L_{C}\end{array} \\
9.80\end{array}$} & \multirow{3}{*}{$\frac{\begin{array}{c}\mathrm{mz}_{\mathrm{z}}^{2} \\
\mathrm{mag}\end{array}}{11.6}$} & \multirow{3}{*}{$\begin{array}{l}\text { Other } \\
\text { Name }\end{array}$} \\
\hline & \multicolumn{6}{|c|}{$(1950)$} & $60 \mu \mathrm{m}$ & $100 \mu \mathrm{m}$ & & & & & \\
\hline & 11 & 44 & 31.9 & -16 & 34 & 26 & 7.7 & 15.4 & & & & & \\
\hline NGC 3893 & 11 & 46 & 0.0 & +48 & 59 & 20 & 14.5 & 34.9 & 977 & $\ldots$ & 10.22 & 10.6 & UGC 6778 \\
\hline NGC 3938 & 11 & 50 & 12.8 & +44 & 23 & 58 & 9.0 & 21.5 & 792 & $\ldots$ & 9.71 & 11.0 & UGC 6856 \\
\hline NGC 3949 & 11 & 51 & 5.0 & +48 & 8 & 13 & 11.2 & 25.0 & 804 & $\ldots$ & 9.89 & 10.9 & UGC 6869 \\
\hline NGC 3953 & 11 & 51 & 11.8 & +52 & 36 & 25 & 7.1 & 36.4 & $\ldots$ & 20.2 & 10.24 & 10.8 & UGC 6870 \\
\hline NGC 3955 & 11 & 51 & 24.2 & -22 & 53 & 10 & 8.4 & 17.4 & 1345 & $\ldots$ & 10.01 & 12.0 & \\
\hline NGC 3981 & 11 & 53 & 35.5 & -19 & 37 & 23 & 6.6 & 18.8 & 1717 & $\ldots$ & 10.22 & 12.5 & Arp 289 \\
\hline NGC 3994 & 11 & 55 & 5.7 & +32 & 34 & 11 & 9.8 & 21.1 & 3250 & $\ldots$ & 10.82 & 12.8 & UGC 6944 \\
\hline NGC 3982 & 11 & 53 & 51.8 & +55 & 24 & 11 & 7.2 & 15.8 & 1110 & $\ldots$ & 9.98 & 12.4 & \\
\hline NGC 4013 & 11 & 55 & 55.9 & +44 & 13 & 34 & 8.4 & 21.6 & 835 & $\ldots$ & 9.84 & 12.4 & \\
\hline NGC 4027 & 11 & 56 & 56.9 & -18 & 59 & 13 & 10.8 & 27.9 & 1677 & $\ldots$ & 10.40 & 11.6 & Arp 22 \\
\hline NGC 4030 & 11 & 57 & 49.4 & -0 & 49 & 16 & 17.5 & 46.4 & 1463 & $\ldots$ & 10.26 & 12.4 & UGC 6993 \\
\hline NGC $4038 / 9$ & 11 & 59 & 19.4 & -18 & 35 & 53 & 41.6 & 76.0 & 1563 & $\ldots$ & 10.81 & 10.5 & Arp 244 \\
\hline NGC 4041 & 11 & 59 & 38.9 & +62 & 24 & 54 & 14.5 & 31.3 & 1234 & $\ldots$ & 10.34 & 11.6 & UGC 7014 \\
\hline NGC 4045 & 12 & 0 & 7.9 & +2 & 15 & 22 & 7.1 & 13.6 & 1995 & $\ldots$ & 10.28 & 13.5 & UGC 7021 \\
\hline NGC 4051 & 12 & 0 & 36.0 & +44 & 48 & 36 & 11.2 & 20.8 & 710 & $\ldots$ & 9.53 & 11.2 & \\
\hline NGC 4085 & 12 & 2 & 49.2 & +50 & 37 & 59 & 5.8 & 14.5 & 751 & $\ldots$ & 9.61 & 12.8 & UGC 7075 \\
\hline NGC 4088 & 12 & 3 & 1.7 & +50 & 49 & 5 & 25.1 & 51.9 & 763 & $\ldots$ & 10.19 & 11.2 & Arp 18 \\
\hline NGC 4096 & 12 & 3 & 28.5 & +47 & 45 & 26 & 7.8 & 19.7 & $\ldots$ & 11.1 & 9.49 & 11.6 & \\
\hline NGC 4100 & 12 & 3 & 36.2 & +49 & 51 & 40 & 9.0 & 20.7 & 1076 & $\ldots$ & 10.08 & 11.7 & UGC 7095 \\
\hline NGC 4102 & 12 & 3 & 50.9 & +52 & 59 & 20 & 49.9 & 67.3 & 863 & $\ldots$ & 10.52 & 11.8 & UGC 7096 \\
\hline NGC 4123 & 12 & 5 & 37.4 & +3 & 9 & 25 & 6.2 & 10.8 & $\ldots$ & 18.0 & 9.68 & 13.1 & UGC 7116 \\
\hline NGC 4157 & 12 & 8 & 34.6 & +50 & 45 & 40 & 19.1 & 43.7 & 771 & $\ldots$ & 10.11 & 11.9 & UGC 7183 \\
\hline NGC 4174 & 12 & 9 & 58.8 & +29 & 26 & 46 & 5.7 & 11.0 & 4061 & $\ldots$ & 10.71 & 14.2 & UGC 7211 \\
\hline IRAS $1211+03$ & 12 & 11 & 12.2 & +3 & 5 & 20 & 8.8 & 9.5 & 21703 & $\ldots$ & 12.19 & $16.9^{1}$ & \\
\hline NGC 4192 & 12 & 11 & 16.1 & +15 & 10 & 34 & 8.8 & 26.4 & $\ldots$ & 12.4 & 9.68 & 11.0 & M98 \\
\hline NGC 4194 & 12 & 11 & 41.3 & +54 & 48 & 11 & 23.4 & 25.0 & 2528 & $\ldots$ & 10.87 & 13.0 & \\
\hline NGC 4212 & 12 & 13 & 2.6 & +14 & 11 & 10 & 7.0 & 16.4 & 2125 & $\ldots$ & 9.79 & 11.9 & UGC 7275 \\
\hline NGC 4214 & 12 & 13 & 9.4 & +36 & 36 & 4 & 16.5 & 29.2 & 288 & $\ldots$ & 8.70 & 10.3 & \\
\hline NGC 4254 & 12 & 16 & 17.3 & +14 & 41 & 38 & 35.2 & 73.2 & 2447 & $\cdots$ & 10.47 & 10.2 & M99 \\
\hline NGC 4273 & 12 & 17 & 22.3 & +5 & 37 & 16 & 10.4 & 21.5 & 2375 & $\ldots$ & 10.60 & 12.3 & UGC 7380 \\
\hline NGC 4298 & 12 & 19 & 3.6 & +14 & 52 & 44 & 6.7 & 19.1 & 1116 & $\ldots$ & 9.85 & 12.1 & UGC 7412 \\
\hline NGC 4303 & 12 & 19 & 24.0 & +4 & 44 & 53 & 35.2 & 61.8 & 1568 & $\ldots$ & 10.85 & 10.9 & M61 \\
\hline NGC 4321 & 12 & 20 & 24.7 & +16 & 5 & 46 & 23.4 & 58.1 & 1576 & $\ldots$ & 10.33 & 10.6 & M100 \\
\hline NGC 4332 & 12 & 20 & 27.1 & +66 & 7 & 12 & 8.0 & 14.0 & 2843 & $\ldots$ & 10.57 & 13.2 & UGC 7453 \\
\hline NGC 4369 & 12 & 22 & 8.2 & +39 & 39 & 32 & 6.3 & 11.3 & 1052 & $\ldots$ & 9.88 & 12.3 & Mrk 439 \\
\hline IRAS $1222-06$ & 12 & 22 & 29.0 & -6 & 24 & 14 & 6.4 & 7.5 & 7495 & $\ldots$ & 11.14 & $\ldots$ & \\
\hline NGC 4383 & 12 & 22 & 53.0 & +16 & 44 & 53 & 9.1 & 12.0 & 1695 & $\ldots$ & 9.77 & 12.3 & UGC 7507 \\
\hline NGC 4388 & 12 & 23 & 14.4 & +12 & 56 & 24 & 11.1 & 17.4 & $\ldots$ & 18.8 & 9.94 & 12.2 & UGC 7520 \\
\hline NGC 4395 & 12 & 23 & 20.0 & +33 & 49 & 30 & 5.7 & 13.4 & 294 & $\ldots$ & 8.29 & 11.7 & \\
\hline NGC 4402 & 12 & 23 & 35.3 & +13 & 23 & 24 & 6.0 & 17.4 & 242 & $\ldots$ & 9.81 & 13.6 & UGC 7528 \\
\hline NGC 4414 & 12 & 23 & 57.8 & +31 & 29 & 56 & 27.6 & 68.6 & $\ldots$ & 13.0 & 10.14 & 10.9 & UGC 7539 \\
\hline NGC 4418 & 12 & 24 & 22.1 & -0 & 36 & 14 & 43.7 & 32.0 & 2045 & $\ldots$ & 11.00 & 14.2 & UGC 7545 \\
\hline NGC 4419 & 12 & 24 & 24.5 & +15 & 19 & 26 & 8.1 & 17.7 & 37 & $\ldots$ & 9.84 & 11.6 & UGC 7551 \\
\hline NGC 4433 & 12 & 25 & 4.6 & -8 & 0 & 14 & 14.1 & 25.6 & 2978 & $\ldots$ & 10.82 & 13.0 & \\
\hline NGC 4438 & 12 & 25 & 14.0 & +13 & 17 & 06 & 5.5 & 15.5 & 259 & $\ldots$ & 9.77 & 12.0 & \\
\hline NGC 4490 & 12 & 28 & 8.2 & +41 & 55 & 23 & 42.5 & 78.1 & 577 & $\ldots$ & 9.80 & 10.1 & UGC 7651 \\
\hline NGC 4501 & 12 & 29 & 28.1 & +14 & 41 & 28 & 16.7 & 56.2 & $\ldots$ & 17.5 & 10.32 & 10.6 & M88 \\
\hline NGC 4526 & 12 & 31 & 30.7 & +7 & 58 & 26 & 6.2 & 15.8 & 447 & $\ldots$ & 9.80 & 10.6 & UGC 7718 \\
\hline NGC 4527 & 12 & 31 & 35.0 & +2 & 55 & 48 & 27.3 & 63.7 & 1737 & $\ldots$ & 10.87 & 12.4 & UGC 7721 \\
\hline
\end{tabular}

magnitudes given in Table $1 \sigma\left[\log \left(L_{b}\right)\right]=0.43$, while $\sigma\left[\log \left(L_{\text {FIR }}\right)\right]=0.70$ for all 324 galaxies in the sample.

The objects in the bright galaxy sample are, not surprisingly, more "infrared active" than those in an optical magnitude limited sample detected in the IRAS survey. This is illustrated in Figure 4, where histograms of far-infrared to blue flux ratios are plotted for the bright galaxy sample and for the galaxies brighter than $14.5 \mathrm{mag}$ in the UGC catalog (Nilson 1973; Rice, private communication) that have $I R A S$ detections.

For the infrared-selected sample the values of $\log \left(f_{\mathrm{FIR}} / f_{b}\right)$ range from -0.9 to 2.1 , while the range for the optically selected sample is -1.5 to 2.1 . The median value of $\log \left(f_{\mathrm{FIR}} / f_{b}\right)$ for the IRAS galaxies is $\sim 0.4$, while for the optical sample the median value is $\sim-0.2$. Note that the UGC galaxies without IRAS detections will have $\log \left(f_{\mathrm{FIR}} / f_{b}\right)<0$. Since only half of the UGC galaxies with $m_{b}<14.5$ mag are detected by $I R A S$, the median value of $\log \left(f_{\mathrm{FIR}} / f_{b}\right)$ for an optically selected sample with infrared measurements for all sources must be still smaller. From Figure 4 it is clear that the infrared flux-limited sample consists of galaxies with much greater average infrared luminosity than does the optically selected sample.

Figure $5 a$ shows that $f_{\text {FIR }} / f_{b}$ correlates with $L_{\text {FIR }}$, while there is no correlation between $f_{\mathrm{FIR}} / f_{b}$ and $L_{b}$, as shown in Figure $5 b$. As seen in Figures 3 and $5 b$ the blue luminosities of the galaxies in the bright galaxy sample have a dispersion of $\sim 1$ mag about a mean of $\sim 10^{10} L_{\odot}\left(M_{b} \approx-20 \mathrm{mag}\right)$ so that larger $f_{\mathrm{FIR}} / f_{b}$ ratios require larger $L_{\mathrm{FIR}}$. The simplest explanation of these results is that the far-infrared and blue luminosity components are basically independent, and the correlation of $f_{\mathrm{FIR}} / f_{b}$ with $L_{\text {FIR }}$ is due to increasing infrared emission in the more lumin- 
TABLE 1-Continued

\begin{tabular}{|c|c|c|c|c|c|c|c|c|c|c|c|c|c|}
\hline \multirow{3}{*}{$\begin{array}{l}\text { NAME } \\
\text { NGC } 4532\end{array}$} & \multicolumn{3}{|c|}{ RA } & \multicolumn{3}{|c|}{ DEC } & \multicolumn{2}{|c|}{$\mathrm{F}_{\nu}(\mathrm{Jy})$} & \multirow{3}{*}{$\begin{array}{c}\mathrm{cz} \\
\mathbf{k m ~ ~ ^ { - 1 }} \\
\ldots\end{array}$} & \multirow{3}{*}{$\begin{array}{c}\begin{array}{c}\mathrm{D} \\
\mathrm{Mpc}\end{array} \\
20.5\end{array}$} & \multirow{3}{*}{$\begin{array}{c}\begin{array}{c}\log \mathrm{L}_{\text {fir }} \\
\mathrm{L}_{\odot}\end{array} \\
9.97\end{array}$} & \multirow{3}{*}{$\begin{array}{r}\begin{array}{r}\mathrm{m}_{\mathrm{z}}{ }^{\mathrm{a}} \\
\mathrm{mag}\end{array} \\
12.3\end{array}$} & \multirow{3}{*}{$\begin{array}{c}\begin{array}{c}\text { Other } \\
\text { Name }\end{array} \\
\text { UGC } 7726\end{array}$} \\
\hline & \multicolumn{6}{|c|}{$(1950)$} & $60 \mu \mathrm{m}$ & $100 \mu \mathrm{m}$ & & & & & \\
\hline & 12 & 31 & 46.3 & +6 & 44 & 38 & 9.5 & 15.3 & & & & & \\
\hline NGC 4535 & 12 & 31 & 48.2 & +8 & 28 & 16 & 9.3 & 24.3 & $\ldots$ & 16.1 & 9.90 & 11.1 & UGC 7727 \\
\hline NGC 4536 & 12 & 31 & 52.6 & +2 & 27 & 58 & 32.0 & 44.1 & 1824 & $\ldots$ & 10.83 & 11.2 & \\
\hline NGC 4559 & 12 & 33 & 29.0 & +28 & 14 & 2 & 11.1 & 28.4 & $\ldots$ & 13.0 & 9.79 & 10.6 & \\
\hline NGC 4565 & 12 & 33 & 52.1 & +26 & 15 & 32 & 11.6 & 48.7 & $\ldots$ & 13.0 & 10.01 & 10.6 & \\
\hline NGC 4568 & 12 & 34 & 2.4 & +11 & 30 & 54 & 20.9 & 47.8 & 2253 & $\ldots$ & 10.26 & 12.5 & UGC 7776 \\
\hline NGC 4569 & 12 & 34 & 18.0 & +13 & 26 & 20 & 10.6 & 28.4 & $\ldots$ & 11.5 & 9.67 & 11.8 & Arp 76 \\
\hline NGC 4579 & 12 & 35 & 11.6 & +12 & 5 & 37 & 6.6 & 17.4 & 1805 & $\ldots$ & 9.83 & 11.5 & M58 \\
\hline NGC 4594 & 12 & 37 & 23.0 & -11 & 21 & 00 & 5.6 & 23.8 & 1128 & $\ldots$ & 10.12 & 9.0 & M104 \\
\hline NGC 4605 & 12 & 37 & 48.7 & +61 & 52 & 52 & 12.9 & 30.3 & 140 & $\ldots$ & 8.80 & 10.8 & UGC 7831 \\
\hline NGC 4618 & 12 & 39 & 7.8 & +41 & 25 & 16 & 6.0 & 11.2 & 558 & $\ldots$ & 8.93 & 11.5 & UGC 7853 \\
\hline NGC 4631 & 12 & 39 & 40.8 & +32 & 49 & 5 & 90.0 & 207.8 & 613 & $\ldots$ & 10.09 & 9.8 & Arp 281 \\
\hline NGC 4651 & 12 & 41 & 13.0 & +16 & 39 & 58 & 5.5 & 15.4 & $\ldots$ & 21.2 & 9.93 & 11.3 & Arp 189 \\
\hline NGC 4654 & 12 & 41 & 25.2 & +13 & 24 & 7 & 13.7 & 35.2 & $\ldots$ & 16.2 & 10.07 & 11.8 & UGC 7902 \\
\hline NGC 4656 & 12 & 41 & 32.0 & +32 & 26 & 30 & 7.2 & 12.3 & 645 & $\ldots$ & 8.95 & 10.6 & \\
\hline NGC 4666 & 12 & 42 & 34.6 & -0 & 11 & 20 & 34.8 & 77.9 & 1645 & $\ldots$ & 10.93 & 12.0 & UGC 7926 \\
\hline NGC 4691 & 12 & 45 & 38.6 & -3 & 3 & 36 & 15.8 & 21.1 & $\ldots$ & 23.8 & 10.28 & 12.0 & \\
\hline NGC 4710 & 12 & 47 & 9.1 & +15 & 26 & 13 & 6.4 & 13.1 & 1125 & $\ldots$ & 9.73 & 11.6 & UGC 7980 \\
\hline $\mathrm{MCG}+08-23-097$ & 12 & 48 & 21.4 & +48 & 12 & 18 & 5.4 & 7.8 & 8833 & $\ldots$ & 11.26 & 16.0 & \\
\hline NGC 4736 & 12 & 48 & 31.7 & +41 & 23 & 35 & 70.0 & 138.7 & 307 & $\ldots$ & 9.52 & 8.7 & M94 \\
\hline NGC 4781 & 12 & 51 & 46.3 & -10 & 15 & 50 & 8.3 & 18.0 & 1265 & $\ldots$ & 10.10 & 12.0 & \\
\hline NGC 4783 & 12 & 52 & 15.8 & +29 & 12 & 36 & 12.1 & 27.8 & 2515 & $\ldots$ & 10.75 & 12.3 & UGC 8033 \\
\hline NGC 4808 & 12 & 53 & 15.8 & +4 & 34 & 34 & 7.1 & 15.0 & $\ldots$ & 16.1 & 9.70 & 12.5 & UGC 8054 \\
\hline IC 3908 & 12 & 54 & 4.1 & -7 & 17 & 24 & 8.8 & 15.9 & 1052 & $\ldots$ & 9.36 & 14.0 & \\
\hline UGC 8058 & 12 & 54 & 4.8 & +57 & 8 & 38 & 33.9 & 29.5 & 12556 & $\ldots$ & 12.32 & 14.1 & Mrk 231 \\
\hline NGC 4818 & 12 & 54 & 12.7 & -8 & 15 & 18 & 20.9 & 25.9 & 1050 & $\ldots$ & 9.67 & 12.0 & \\
\hline NGC 4826 & 12 & 54 & 17.5 & +21 & 57 & 7 & 30.2 & 78.7 & 414 & $\ldots$ & 9.24 & 8.9 & M64 \\
\hline NGC 4845 & 12 & 55 & 27.8 & +1 & 50 & 42 & 9.9 & 23.5 & $\ldots$ & 17.4 & 9.94 & 12.9 & UGC 8078 \\
\hline NGC 4900 & 12 & 58 & 5.8 & +2 & 46 & 12 & 5.8 & 12.1 & $\ldots$ & 17.7 & 9.69 & 12.8 & UGC 8116 \\
\hline NGC 4922 & 12 & 59 & 1.0 & +29 & 34 & 59 & 6.7 & 6.7 & 7357 & $\ldots$ & 11.14 & 14.2 & UGC 8135 \\
\hline MCG+01-33-036 & 12 & 59 & 17.8 & +4 & 36 & 4 & 5.6 & 7.4 & 10852 & $\ldots$ & 11.42 & 15.5 & \\
\hline NGC 5005 & 13 & 8 & 37.9 & +37 & 19 & 26 & 19.6 & 59.9 & 950 & $\ldots$ & 10.50 & 10.6 & UGC 8256 \\
\hline NGC 5020 & 13 & 10 & 12.5 & +12 & 51 & 40 & 5.4 & 9.9 & 3354 & $\ldots$ & 10.52 & 13.4 & UGC 8289 \\
\hline NGC 5033 & 13 & 11 & 9.8 & +36 & 51 & 25 & 19.5 & 53.0 & 877 & $\ldots$ & 10.40 & 10.9 & UGC 8307 \\
\hline IC 860 & 13 & 12 & 40.1 & +24 & 52 & 52 & 18.4 & 17.9 & 3862 & $\ldots$ & 11.10 & 14.8 & \\
\hline NGC 5055 & 13 & 13 & 34.8 & +42 & 17 & 31 & 45.3 & 161.0 & 497 & $\ldots$ & 10.01 & 9.7 & M63 \\
\hline UGC 8335 & 13 & 13 & 41.3 & +62 & 23 & 17 & 11.5 & 10.2 & 9356 & $\ldots$ & 11.60 & 14.4 & VII Zw 506 \\
\hline NGC 5073 & 13 & 16 & 42.5 & -14 & 35 & 6 & 9.5 & 14.8 & 2715 & $\ldots$ & 10.54 & 13.0 & \\
\hline UGC 8387 & 13 & 18 & 19.0 & +34 & 23 & 49 & 16.0 & 23.8 & 6870 & $\ldots$ & 11.52 & 14.8 & Arp 193 \\
\hline NGC 5104 & 13 & 18 & 49.2 & +0 & 36 & 14 & 7.5 & 12.5 & 5585 & $\ldots$ & 11.04 & 14.5 & UGC 8391 \\
\hline NGC 5145 & 13 & 23 & 3.8 & +43 & 31 & 26 & 6.7 & 12.0 & 1225 & $\ldots$ & 9.99 & 13.6 & UGC 8439 \\
\hline NGC 5194 & 13 & 27 & 45.4 & +47 & 27 & 25 & 121.0 & 299.0 & $\ldots$ & 9.3 & 10.49 & 8.8 & M51 \\
\hline NGC 5195 & 13 & 27 & 52.8 & +47 & 31 & 30 & 17.0 & 20.0 & $\ldots$ & 9.3 & 9.47 & 10.6 & UGC 8494 \\
\hline NGC 5218 & 13 & 30 & 26.4 & +63 & 1 & 26 & 7.6 & 14.2 & 2860 & $\ldots$ & 10.57 & 13.1 & \\
\hline NGC 5248 & 13 & 35 & 2.6 & +9 & 8 & 28 & 18.6 & 43.9 & 1156 & $\ldots$ & 10.22 & 11.4 & UGC 8616 \\
\hline NGC 5256 & 13 & 36 & 14.2 & +48 & 31 & 52 & 7.7 & 11.9 & 8285 & $\ldots$ & 11.37 & 14.1 & UGC 8632 \\
\hline NGC $5257 / 8$ & 13 & 37 & 22.1 & +1 & 5 & 13 & 11.0 & 18.3 & 6820 & $\ldots$ & 11.37 & 13.7 & UGC $8641 / 5$ \\
\hline UGC 8696 & 13 & 42 & 51.6 & +56 & 8 & 13 & 24.5 & 21.2 & 11400 & $\ldots$ & 12.10 & 15.0 & Mrk 273 \\
\hline UGC 8739 & 13 & 47 & 1.7 & +35 & 30 & 14 & 6.4 & 14.3 & 5130 & $\ldots$ & 11.00 & 14.7 & \\
\hline NGC 5331 & 13 & 49 & 41.3 & +2 & 21 & 7 & 6.0 & 10.2 & 9950 & $\ldots$ & 11.43 & 14.3 & VV253 \\
\hline
\end{tabular}

ous galaxies, rather than due to extinction of the visible radiation.

The bright galaxy sample contains no galaxies with low farinfrared luminosities and with $f_{\mathrm{FIR}} / f_{b}$ ratios greater than 10 . This lack cannot be a selection effect. At $L_{\text {FIR }} \approx 10^{10} L_{\odot}$ the bright galaxy sample includes galaxies to $30 \mathrm{Mpc}$, certainly a large enough volume to detect such galaxies if they were common. Even at $L_{\text {FIR }} \approx 10^{9} L_{\odot}$, galaxies would be detected to $\sim 10 \mathrm{Mpc}$. Such galaxies might have very low visible surface brightness and hence not be visible on the POSS. Such galaxies would, if their sizes were similar to the optical size of dwarf irregular galaxies (Gallagher and Hunter 1984), be point sources at distances greater than $6 \mathrm{Mpc}$, so that they would be contained in the PSC for over $80 \%$ of the volume surveyed. Thus the identifications from this subset of the bright galaxy sample, where only one object is not accounted for (see above), preclude a significant contribution of dwarf galaxies. The lack of any visible, faint galaxy counterparts to extended sources also argues against such a class of galaxies being present in the SSSC. The work of Helou (1986a) has shown that a very small fraction of known dwarf galaxies have detectable $60 \mu \mathrm{m}$ emission, and this is at a comparatively low level and usually associated with $\mathrm{H}$ II regions in these galaxies.

Seven galaxies in the bright galaxy sample are contained in the blue compact galaxy sample of Thuan and Martin (1981). The average absolute blue magnitude of these seven galaxies is $M_{b}=-19.9 \mathrm{mag}$. The mean far-infrared luminosity of these galaxies is $1.8 \times 10^{10} L_{\odot}$, and their mean ratio of infrared to blue light is 2.5 ; all of these values are close to the median of the entire sample. Thus the infrared properties of the blue 
TABLE 1-Continued

\begin{tabular}{|c|c|c|c|c|c|c|c|c|c|c|c|c|c|}
\hline \multirow{3}{*}{$\begin{array}{l}\text { NAME } \\
\text { NGC } 5371\end{array}$} & \multicolumn{3}{|c|}{ RA } & \multicolumn{3}{|c|}{ DEC } & \multicolumn{2}{|c|}{$\mathrm{F}_{\nu}(\mathrm{Jy})$} & \multirow{3}{*}{$\frac{\begin{array}{c}\mathrm{cz}^{-1} \\
\mathrm{~km} \mathrm{~s}^{-1}\end{array}}{2565}$} & \multirow{3}{*}{$\begin{array}{c}\begin{array}{c}\mathrm{D} \\
\mathrm{Mpc}\end{array} \\
\ldots\end{array}$} & \multirow{3}{*}{$\begin{array}{c}\log _{\text {fir }} \\
L_{(}\end{array}$} & \multirow{3}{*}{$\begin{array}{r}\begin{array}{c}\mathrm{m}_{\mathrm{z}}^{2} \\
\mathrm{mag}\end{array} \\
11.5\end{array}$} & \multirow{3}{*}{$\begin{array}{c}\begin{array}{c}\text { Other } \\
\text { Name }\end{array} \\
\text { UGC } 8846\end{array}$} \\
\hline & & & & & & & \multirow{2}{*}{$\begin{array}{c}60 \mu \mathrm{m} \\
5.7\end{array}$} & \multirow{2}{*}{$\begin{array}{c}100 \mu \mathrm{m} \\
14.0\end{array}$} & & & & & \\
\hline & 13 & 53 & 32.5 & +40 & 42 & 13 & & & & & & & \\
\hline NGC 5383 & 13 & 55 & 0.2 & +42 & 5 & 20 & 5.5 & 12.9 & 2282 & $\ldots$ & 10.35 & 12.5 & UGC 8875 \\
\hline NGC 5394 & 13 & 56 & 25.2 & +37 & 41 & 38 & 10.1 & 11.9 & 3404 & $\ldots$ & 10.74 & 13.9 & \\
\hline NGC 5430 & 13 & 59 & 8.4 & +59 & 34 & 12 & 10.9 & 20.2 & 2819 & $\ldots$ & 10.72 & 13.1 & \\
\hline NGC 5433 & 14 & 0 & 24.0 & +32 & 45 & 0 & 7.2 & 11.1 & 4278 & $\ldots$ & 10.81 & 14.0 & UGC 8954 \\
\hline NGC 5427 & 14 & 0 & 48.3 & -5 & 47 & 25 & 9.1 & 27.1 & 2565 & $\ldots$ & 10.70 & 12.0 & Arp 271 \\
\hline NGC 5457 & 14 & 1 & 55.7 & +54 & 33 & 22 & 96.7 & 257.4 & $\ldots$ & 8.1 & 10.32 & 8.7 & M101 \\
\hline NGC 5506 & 14 & 10 & 38.9 & -2 & 58 & 26 & 8.8 & 9.3 & 1809 & $\ldots$ & 10.20 & 13.6 & \\
\hline Zw 247.020 & 14 & 17 & 53.8 & +49 & 27 & 54 & 6.5 & 8.1 & 7800 & $\therefore$ & 11.21 & 15.4 & Mrk 1490 \\
\hline NGC 5600 & 14 & 21 & 25.7 & +14 & 51 & 54 & 5.9 & 11.4 & 2349 & $\ldots$ & 10.33 & 11.9 & UGC 9220 \\
\hline NGC 5595 & 14 & 21 & 27.1 & -16 & 29 & 53 & 8.9 & 15.8 & 2691 & $\ldots$ & 10.53 & 12.5 & \\
\hline NGC 5597 & 14 & 21 & 41.0 & -16 & 32 & 10 & 9.1 & 15.1 & 2677 & $\ldots$ & 10.53 & 13.0 & \\
\hline NGC 5653 & 14 & 28 & 0.2 & +31 & 26 & 17 & 11.5 & 20.8 & 3514 & $\ldots$ & 10.90 & 12.7 & UGC 9318 \\
\hline NGC 5663 & 14 & 29 & 57.4 & +8 & 18 & 0 & 6.9 & 12.8 & 2266 & $\ldots$ & 10.35 & 12.6 & UGC 9352 \\
\hline NGC 5678 & 14 & 30 & 37.4 & +58 & 8 & 17 & 8.9 & 25.3 & 1929 & $\ldots$ & 10.52 & 12.3 & \\
\hline NGC 5676 & 14 & 31 & 1.2 & +49 & 40 & 37 & 10.8 & 30.6 & 2104 & $\ldots$ & 10.67 & 11.7 & UGC 9366 \\
\hline IRAS $1434-14$ & 14 & 34 & 52.3 & -14 & 47 & 24 & 7.1 & 7.2 & 24332 & $\ldots$ & 12.19 & $16.7^{1}$ & \\
\hline NGC 5690 & 14 & 35 & 8.4 & +2 & 30 & 25 & 6.8 & 16.1 & 1750 & $\ldots$ & 10.24 & 13.1 & \\
\hline NGC 5713 & 14 & 37 & 37.2 & -0 & 4 & 34 & 20.9 & 36.9 & 1900 & $\ldots$ & 10.69 & 11.7 & UGC 9451 \\
\hline NGC 5719 & 14 & 38 & 22.6 & -0 & 6 & 18 & 8.7 & 17.1 & 1480 & $\ldots$ & 10.18 & 13.8 & UGC 9462 \\
\hline NGC 5728 & 14 & 39 & 39.4 & -17 & 2 & 42 & 8.8 & 14.9 & 2813 & $\ldots$ & 10.55 & 12.5 & VV75 \\
\hline NGC 5757 & 14 & 44 & 57.8 & -18 & 52 & 16 & 6.4 & 13.0 & 2771 & $\ldots$ & 10.46 & 12.5 & \\
\hline NGC 5775 & 14 & 51 & 26.9 & +3 & 44 & 38 & 24.2 & 45.3 & 1670 & $\ldots$ & 10.69 & 13.0 & UGC 9579 \\
\hline UGC 9618 & 14 & 54 & 47.8 & +24 & 48 & 58 & 6.8 & 15.3 & 10100 & $\ldots$ & 11.59 & 14.3 & Arp 302 \\
\hline NGC 5792 & 14 & 55 & 46.6 & -0 & 53 & 24 & 9.5 & 19.1 & 1930 & $\ldots$ & 10.40 & 13.5 & UGC 9631 \\
\hline NGC 5793 & 14 & 56 & 39.6 & -16 & 29 & 53 & 6.8 & 9.9 & 3521 & $\ldots$ & 10.58 & 14.0 & \\
\hline UGC 9668 & 15 & 0 & 33.8 & +83 & 43 & 19 & 5.4 & 7.6 & 3917 & $\ldots$ & 10.59 & 13.8 & Mrk 839 \\
\hline NGC 5866 & 15 & 5 & 7.2 & +55 & 57 & 14 & 5.4 & 17.0 & 672 & $\ldots$ & 9.66 & 11.1 & UGC 9723 \\
\hline NGC 5861 & 15 & 6 & 33.1 & -11 & 7 & 59 & 10.9 & 20.6 & 1867 & $\ldots$ & 10.38 & 12.0 & \\
\hline Zw 049.057 & 15 & 10 & 45.6 & +7 & 24 & 43 & 23.1 & 30.6 & 3528 & $\ldots$ & 11.12 & 15.5 & \\
\hline NGC 5900 & 15 & 13 & 17.0 & +42 & 23 & 35 & 8.2 & 16.0 & 2551 & $\ldots$ & 10.54 & 15.0 & UGC 9790 \\
\hline NGC 5907 & 15 & 14 & 40.8 & +56 & 29 & 35 & 11.2 & 50.2 & 666 & $\ldots$ & 10.16 & 11.4 & UGC 9801 \\
\hline I Zw 107 & 15 & 16 & 19.0 & +42 & 55 & 41 & 9.7 & 9.8 & 12043 & $\ldots$ & 11.72 & 14.9 & Mrk 848 \\
\hline NGC 5915 & 15 & 18 & 47.5 & -12 & 54 & 50 & 11.3 & 15.6 & 2338 & $\ldots$ & 10.48 & 12.5 & \\
\hline NGC 5929 & 15 & 24 & 20.6 & +41 & 50 & 56 & 9.8 & 13.3 & 2600 & $\ldots$ & 10.55 & 13.0 & UGC 9852 \\
\hline IRAS $1525+36$ & 15 & 25 & 3.1 & +36 & 9 & 0 & 7.5 & 5.4 & 16009 & $\ldots$ & 11.89 & $16.2^{1}$ & \\
\hline NGC 5936 & 15 & 27 & 39.4 & +13 & 9 & 32 & 9.3 & 16.4 & 4029 & $\ldots$ & 10.89 & 13.0 & UGC 9867 \\
\hline NGC 5937 & 15 & 28 & 9.8 & -2 & 39 & 36 & 10.5 & 21.0 & 2488 & $\ldots$ & 10.61 & 13.1 & \\
\hline NGC 5953 & 15 & 32 & 13.4 & +15 & 21 & 43 & 11.0 & 20.1 & 1983 & $\ldots$ & 10.46 & 13.3 & Arp 91 \\
\hline UGC 9913 & 15 & 32 & 46.3 & +23 & 40 & 8 & 110.1 & 115.1 & 5452 & $\ldots$ & 12.12 & 14.4 & Arp 220 \\
\hline IRAS $1533-05$ & 15 & 33 & 32.4 & -5 & 13 & 59 & 5.7 & 9.6 & 7800 & $\cdots$ & 11.20 & $16.8^{1}$ & \\
\hline NGC 5962 & 15 & 34 & 13.9 & +16 & 46 & 16 & 9.0 & 21.8 & 1963 & $\ldots$ & 10.45 & 12.2 & UGC 9926 \\
\hline NGC 5990 & 15 & 43 & 44.6 & +2 & 34 & 12 & 10.3 & 15.4 & 3809 & $\ldots$ & 10.84 & 13.1 & UGC 10024 \\
\hline NGC 6015 & 15 & 50 & 39.3 & +62 & 27 & 27 & 6.2 & 10.4 & $\ldots$ & 16.5 & 9.60 & 11.6 & \\
\hline NGC 6052 & 16 & 3 & 2.6 & +20 & 40 & 34 & 7.4 & 9.7 & 4762 & $\ldots$ & 10.87 & 14.1 & Arp 209 \\
\hline NGC 6070 & 16 & 7 & 26.0 & $+\infty$ & 50 & 19 & 7.4 & 12.9 & $\ldots$ & 29.6 & 10.19 & 13.0 & UGC 10230 \\
\hline NGC 6090 & 16 & 10 & 24.0 & +52 & 35 & 6 & 6.8 & 9.6 & 8733 & $\ldots$ & 11.35 & 14.0 & UGC 10267 \\
\hline MCG $+01-42-088$ & 16 & 28 & 27.4 & +4 & 11 & 24 & 7.4 & 11.5 & 7075 & $\ldots$ & 11.21 & 14.9 & \\
\hline NGC 6181 & 16 & 30 & 10.1 & +19 & 55 & 48 & 9.3 & 20.3 & 2379 & $\ldots$ & 10.57 & 12.7 & \\
\hline
\end{tabular}

compact galaxies in the sample are indistinguishable from the properties of the sample as a whole. None of these galaxies meet Thuan and Martin's definition of being dwarfs, i.e., having $M_{B}>-18 \mathrm{mag}$.

In Figure $6 a$ the $60 \mu \mathrm{m} / 100 \mu \mathrm{m}$ flux density ratio, which is monotonic with color temperature, is plotted versus farinfrared luminosity, while in Figure $6 b$ the $60 \mu \mathrm{m} / 100 \mu \mathrm{m}$ flux density ratio is plotted versus blue luminosity. There is a correlation between the color temperature and the far-infrared luminosity in the sense that higher luminosities correspond to higher $60 \mu \mathrm{m} / 100 \mu \mathrm{m}$ color temperatures, while there is clearly no correlation between the far-infrared color temperature and the blue luminosity. Such a correlation has been found previously by Miley, Neugebauer, and Soifer (1985) and Rieke and Lebofsky (1986).
The absence of high-luminosity, cold galaxies from the bright galaxy sample is probably not a selection effect. Cold galaxies of a given far-infrared luminosity will have weaker $60 \mu$ m fluxes than do warm galaxies, so the volume within which they can be detected at $60 \mu \mathrm{m}$ is smaller. This selection effect does not account for the change in color temperature with luminosity and does not appear to be able to account for the observed lack of cold galaxies at high luminosity. The volume searched for galaxies having the median $60 \mu \mathrm{m} /$ $100 \mu$ m color of those galaxies with $L_{\text {FIR }} \approx 10^{10} L_{\odot}$ is $\sim \frac{2}{3}$ that of those galaxies with median color at $L_{\mathrm{FIR}} \approx 10^{12} L_{\odot}$ at the same luminosity. Therefore the lack of detection of any such galaxies cannot be simply a color selection effect, but rather indicates a significant decrease in the space density of galaxies at high luminosities and cold color temperatures. Furthermore, 
TABLE $1-$ Continued

\begin{tabular}{|c|c|c|c|c|c|c|c|c|c|c|c|c|c|}
\hline \multirow[t]{2}{*}{ NAME } & \multicolumn{3}{|c|}{$\mathrm{RA}$} & \multirow{2}{*}{\multicolumn{3}{|c|}{ DEC }} & \multicolumn{2}{|c|}{$\mathrm{F}_{u}(\mathrm{Jy})$} & \multirow{2}{*}{$\begin{array}{c}\mathrm{cz}^{-1} \\
\mathrm{~km} \mathrm{~s}^{-1}\end{array}$} & \multirow{2}{*}{$\begin{array}{c}\mathrm{D} \\
\mathrm{Mpc}\end{array}$} & \multirow{2}{*}{$\begin{array}{c}\log L_{\text {fir }} \\
L_{C} \\
\end{array}$} & \multirow{2}{*}{$\begin{array}{r}\mathrm{m}_{\mathrm{z}}{ }^{2} \\
\mathrm{mag}\end{array}$} & \multirow{2}{*}{$\begin{array}{l}\text { Other } \\
\text { Name } \\
\end{array}$} \\
\hline & & & & & & & $60 \mu \mathrm{m}$ & $100 \mu \mathrm{m}$ & & & & & \\
\hline NGC 6217 & 16 & 35 & 4.8 & +78 & 18 & 4 & 11.0 & 20.9 & 1359 & $\ldots$ & 10.22 & 12.1 & Arp 185 \\
\hline NGC $6285 / 6$ & 16 & 57 & 44.9 & +59 & 0 & 40 & 10.2 & 23.5 & 5600 & $\ldots$ & 11.28 & 14.2 & Arp 293 \\
\hline IRAS $1713+53$ & 17 & 13 & 14.2 & +53 & 13 & 52 & 6.6 & 7.6 & 15212 & $\ldots$ & 11.77 & $16.1^{1}$ & \\
\hline NGC 6503 & 17 & 49 & 57.8 & +70 & 9 & 25 & 12.4 & 25.4 & 51 & $\ldots$ & 8.97 & 10.9 & UGC 11012 \\
\hline MCG-03-57-017 & 22 & 28 & 42.7 & -19 & 17 & 31 & 6.1 & 10.3 & 7263 & $\ldots$ & 11.13 & 14.5 & \\
\hline IRAS 2249-18 & 22 & 49 & 9.6 & -18 & 8 & 20 & 5.6 & 4.3 & 22807 & $\ldots$ & 12.06 & $16.5^{1}$ & \\
\hline NGC 7448 & 22 & 57 & 34.8 & +15 & 42 & 47 & 8.2 & 17.9 & 2192 & $\ldots$ & 10.33 & 12.0 & Arp 13 \\
\hline NGC 7465 & 22 & 59 & 31.9 & +15 & 41 & 55 & 6.8 & 6.5 & 1959 & $\ldots$ & 10.03 & 13.3 & \\
\hline NGC 7469 & 23 & 0 & 44.6 & +8 & 36 & 18 & 27.8 & 34.4 & 4963 & $\ldots$ & 11.40 & 13.0 & UGC 12332 \\
\hline NGC 7479 & 23 & 2 & 26.6 & +12 & 3 & 11 & 12.4 & 24.8 & 2382 & $\cdots$ & 10.55 & 11.7 & UGC 12343 \\
\hline $\mathrm{Zw} 453.062$ & 23 & 2 & 28.1 & +19 & 16 & 55 & 8.0 & 10.7 & 7373 & $\ldots$ & 11.23 & 15.2 & \\
\hline NGC 7541 & 23 & 12 & 11.5 & +4 & 15 & 40 & 19.5 & 39.9 & 2665 & $\ldots$ & 10.83 & 12.7 & UGC 12447 \\
\hline Zw 475.056 & 23 & 13 & 31.2 & +25 & 16 & 48 & 10.1 & 12.1 & 8215 & $\ldots$ & 11.41 & 15.0 & \\
\hline NGC 7591 & 23 & 15 & 43.9 & +6 & 18 & 47 & 8.1 & 13.1 & 4964 & $\ldots$ & 10.92 & 13.8 & UGC 12486 \\
\hline NGC 7592 & 23 & 15 & 47.5 & -4 & 41 & 20 & 8.4 & 10.4 & 7314 & $\ldots$ & 11.21 & 14.0 & \\
\hline NGC 7625 & 23 & 17 & 59.5 & +16 & 57 & 4 & 9.6 & 18.7 & 1653 & $\ldots$ & 10.12 & 12.8 & UGC 12529 \\
\hline NGC 7673 & 23 & 25 & 12.0 & +23 & 18 & 54 & 5.5 & 6.7 & 3407 & $\ldots$ & 10.39 & 12.7 & Mrk 325 \\
\hline NGC 7674 & 23 & 25 & 24.7 & +8 & 30 & 14 & 5.7 & 8.2 & 8669 & $\ldots$ & 11.22 & 13.6 & UGC 12608 \\
\hline NGC 7678 & 23 & 25 & 56.6 & +22 & 8 & 31 & 7.5 & 14.8 & 3491 & $\ldots$ & 10.64 & 12.7 & UGC 12614 \\
\hline NGC 7679 & 23 & 26 & 13.9 & +3 & 14 & 13 & 7.7 & 9.5 & 5152 & $\ldots$ & 10.87 & 13.2 & UGC 12618 \\
\hline NGC 7714 & 23 & 33 & 39.8 & +1 & 52 & 34 & 11.3 & 10.8 & 2805 & $\ldots$ & 10.51 & 13.1 & UGC 12699 \\
\hline NGC 7771 & 23 & 48 & 52.1 & +19 & 49 & 55 & 19.1 & 38.7 & 4346 & $\ldots$ & 11.25 & 13.1 & \\
\hline Mrk 331 & 23 & 48 & 52.8 & +20 & 18 & 22 & 17.6 & 20.3 & 5385 & $\ldots$ & 11.27 & 14.9 & \\
\hline UGC $12915 / 4$ & 23 & 59 & 7.7 & +23 & 12 & 58 & 5.8 & 14.1 & 4590 & $\ldots$ & 10.82 & 13.2 & III $\mathrm{Zw} 125$ \\
\hline
\end{tabular}

a Magnitude taken from Zwicky catalogs (Zwicky et al. 1961-1968). Note (1) indicates magnitude is blue magnitude, from Sanders et al. $1987 a$.

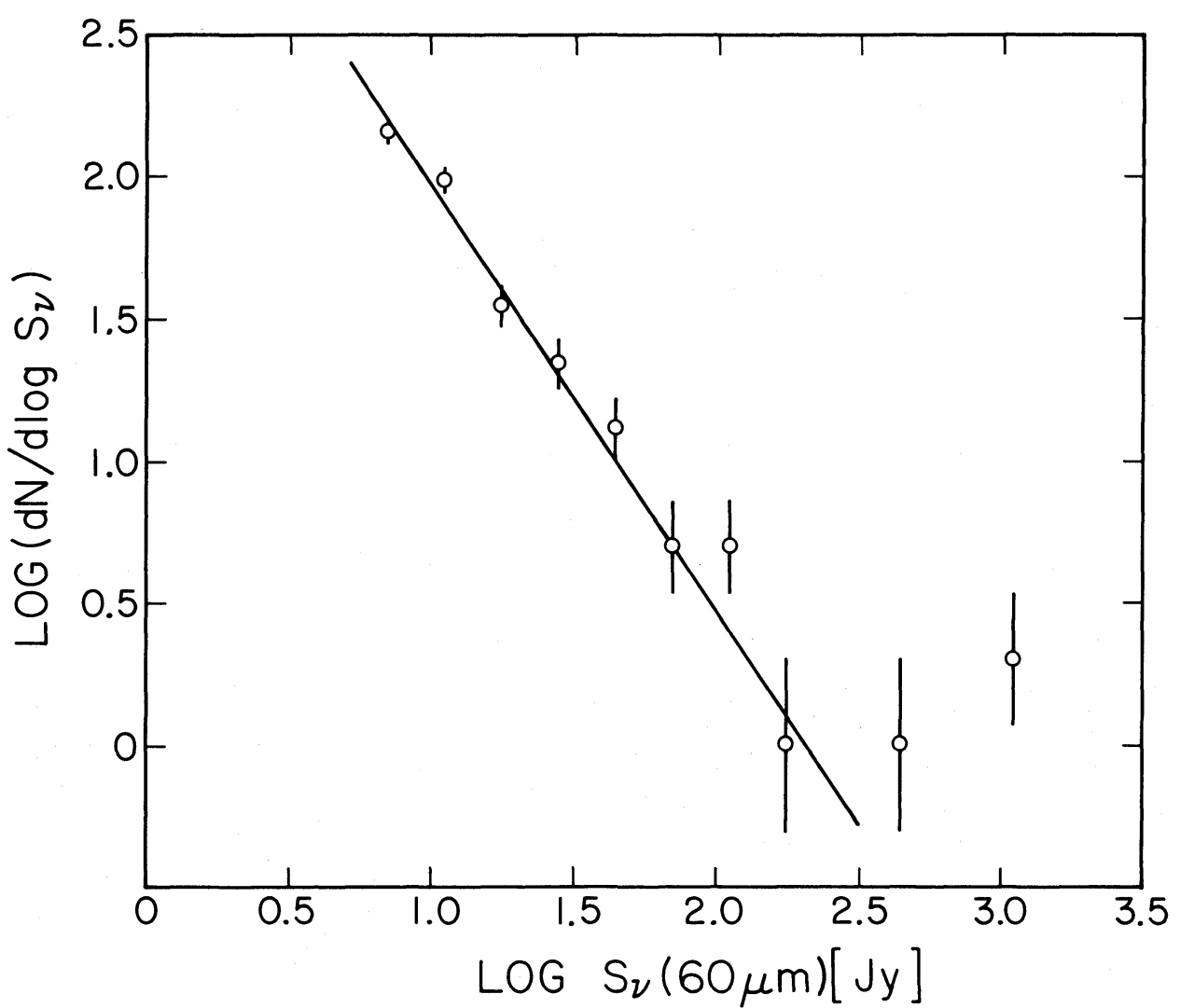

Fig. 1.-Differential number counts of sources plotted vs. flux density for sources in the bright galaxy sample. Each bin includes a range of 1.67 in flux density, while fractional error in each bin is $N^{-1 / 2}$, where $N$ is number of sources in bin. Line shown is best-fit of data to $N \propto f_{v}^{-3 / 2}$ power-law number count distribution, and is an acceptable fit to data. 


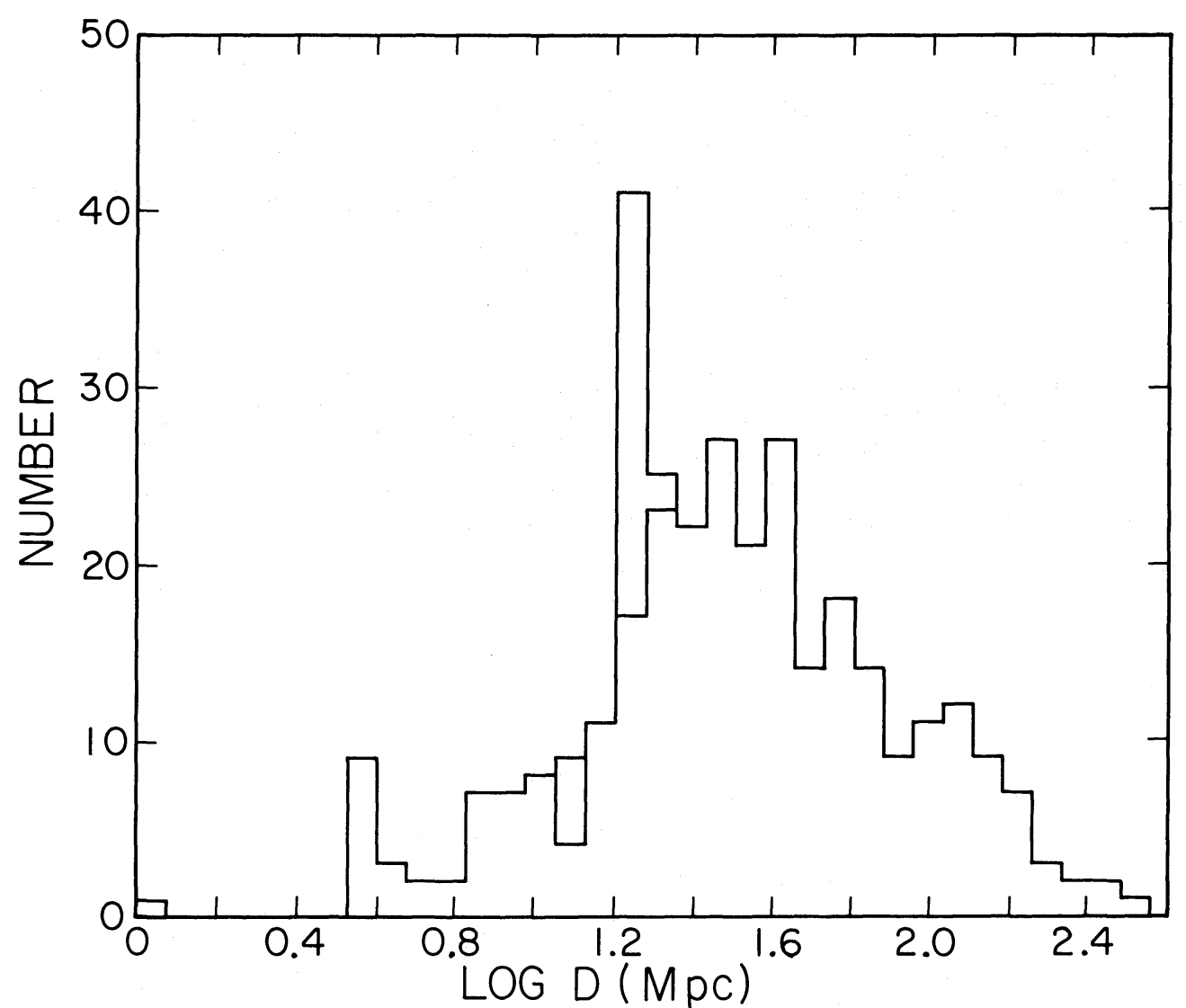

Fig. 2.-Histogram of distances to galaxies in bright galaxy sample, determined as described in text. Lower envelope represents galaxies not associated with Virgo cluster, while histogram above this line includes Virgo cluster galaxies.

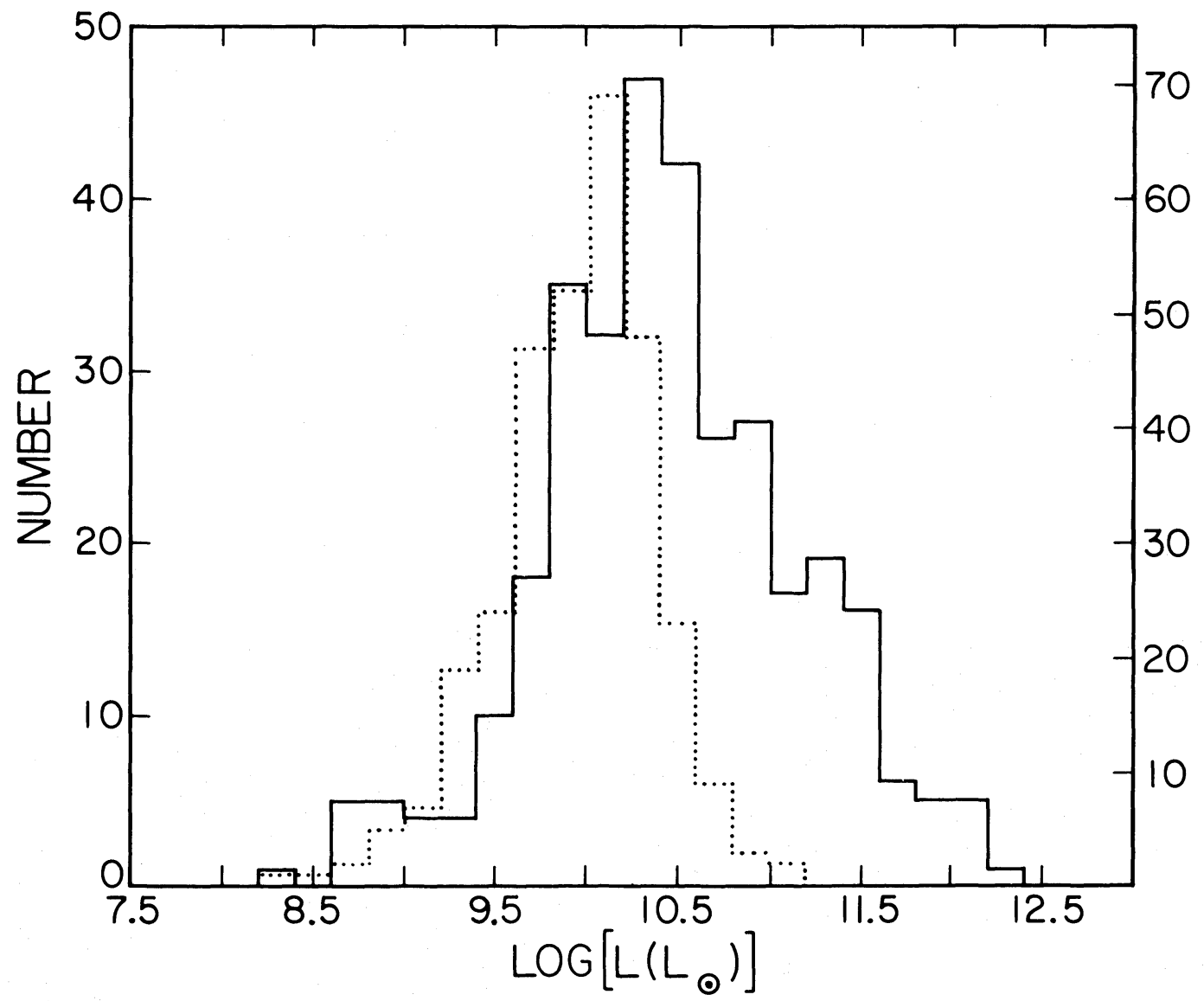

FIG. 3.-Histograms of luminosities of galaxies in bright galaxy sample. Blue luminosity (dotted line) is $v L_{v}(0.43 \mu \mathrm{m})$, while far-infrared luminosity (solid line) is the luminosity effectively from 40-400 $\mu \mathrm{m}$ (see text). In plot and in text luminosities are given in solar (bolometric) luminosities. Note much narrower distribution of blue compared to far-infrared luminosity. 


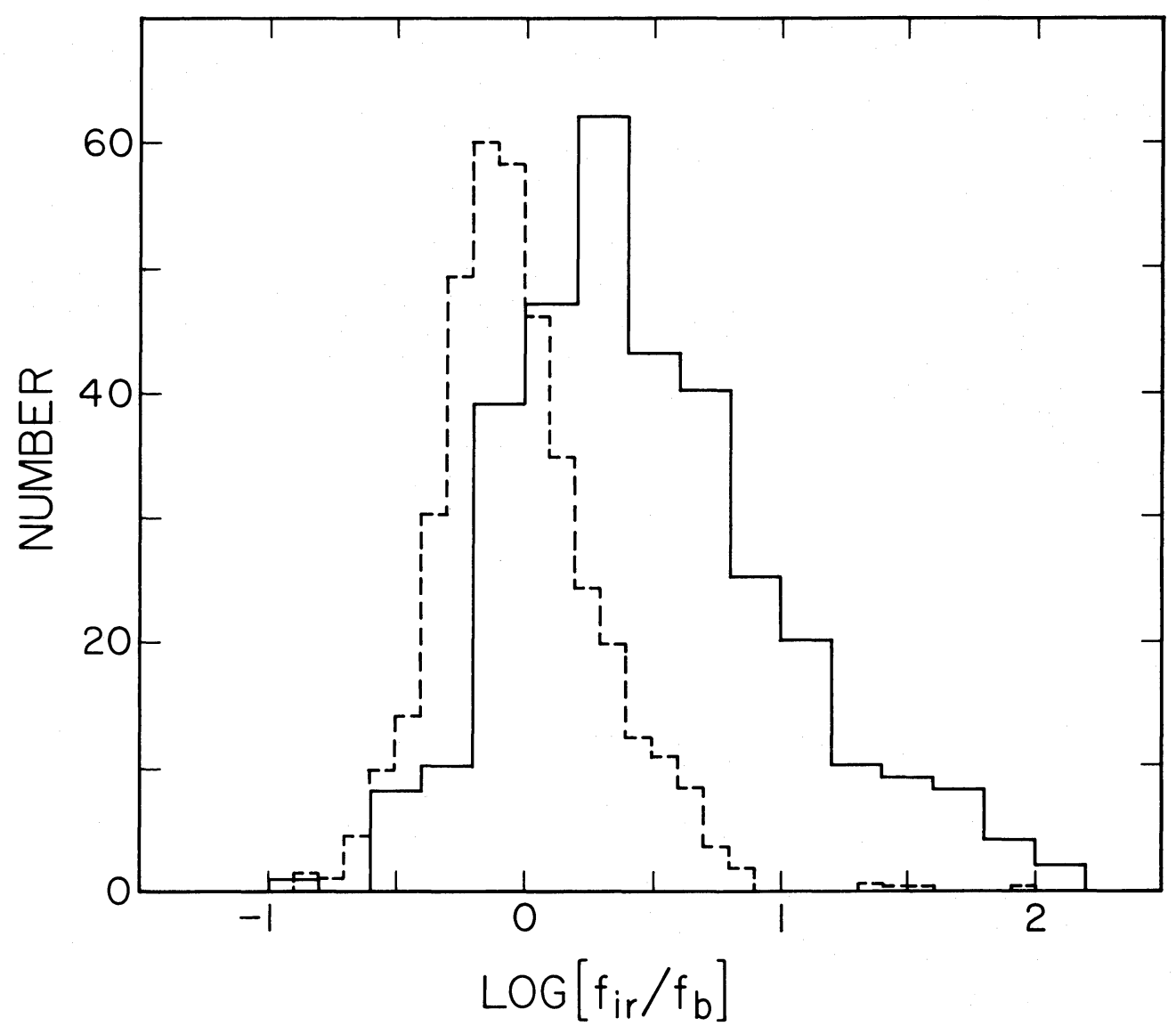

Fig. 4.- Histograms of ratio of far-infrared flux to blue flux for two samples of galaxies detected in IRAS survey. Solid line is infrared limited bright galaxy sample, while dashed line is distribution for galaxies in UGC catalog with $m_{z}<14.5$ detected in IRAS survey (Bothun, Lonsdale, and Rice 1987). Nondetections in UGC catalog have $\log f_{\mathrm{FIR}} / f_{b}<0$. Histogram for UGC galaxies has been normalized to peak of bright galaxy sample, but contains $\sim 10$ times more galaxies. One object from UGC galaxies at $\log f_{\text {FIR }} / f_{b}=-1.55$ fell below limits of plot.

a search of the PSC at $100 \mu \mathrm{m}$ with $b>50^{\circ}$ showed no highluminosity, cold objects that were not contained in the bright galaxy sample.

The apparent maximum $60 \mu \mathrm{m} / 100 \mu \mathrm{m}$ flux density ratio in Figure $6 a$, independent of $L_{\mathrm{FIR}}$, implies that the intensity of the radiation field heating the radiating material reaches an effective maximum. The increase of the lower bound of $60 \mu \mathrm{m} /$ $100 \mu \mathrm{m}$ flux density ratio with increasing luminosity indicates that the minimum radiation field seen by the radiating material is increasing with luminosity.

Lines of constant mass of radiating dust (assuming optically thin dust emission) corresponding to total gas masses (assuming $M_{g} / M_{d}=200$ ) of $10^{8}, 10^{9}$, and $10^{10} M_{\odot}$ are shown in Figure $6 a$. They show that the amount of material responsible for the far-infrared radiation is roughly comparable to the amount of interstellar matter in normal galaxies, and generally increases with increasing luminosity. Nearly all the galaxies in the bright galaxy sample have masses of dust within this range, with a tendency for the higher luminosity sources to have more radiating material. This range of mass is quite comparable to the amount of mass expected in the interstellar medium of normal spiral galaxies (Sanders et al. 1986). Since cold galaxies need more material to produce a given luminosity, the absence of galaxies with high luminosities and low color temperatures may reflect the absence of galaxies having enough interstellar matter to produce such high luminosities without having a generally warmer interstellar medium. Another statement of this is that a luminosity of $10^{12} L_{\odot}$ is sufficient to heat the dust corresponding to more than $10^{10}$ $M_{\odot}$ of gas and dust to temperatures significantly greater than those found in normal galaxies.

Figure 7 combines the previous two figures, showing the ratio $f_{\text {FIR }} / f_{b}$ plotted versus $f_{v}(60 \mu \mathrm{m}) / f_{v}(100 \mu \mathrm{m})$ for the bright galaxy sample. This plot shows the same general correlation shown previously by de Jong et al. (1984) and Soifer et al. (1984), where increasing ratio of infrared to blue light is correlated with increasing color temperature. At a given $60 \mu \mathrm{m} /$ $100 \mu \mathrm{m}$ ratio, the spread in $f_{\text {FIR }} / f_{b}$ is greater in the bright galaxy sample than in the optically selected sample of de Jong et al., while the lower envelope of the $f_{\mathrm{FIR}} / f_{b}$ versus $f_{v}(60 \mu \mathrm{m}) /$ $f_{v}(100 \mu \mathrm{m})$ relation is consistent with the results from the optically selected sample.

\section{SPACE DENSITY OF IR $A S$ GALAXIES}

a) The $60 \mu \mathrm{m}$ Luminosity Function

The space density of galaxies in terms of $60 \mu \mathrm{m}$ luminosity, $v L_{v}(60 \mu \mathrm{m})$, and the uncertainty in the space density were derived using the expressions

$$
\rho=\left(\frac{4 \pi}{\Omega}\right)\left(\sum \frac{1}{V_{m}}\right),
$$




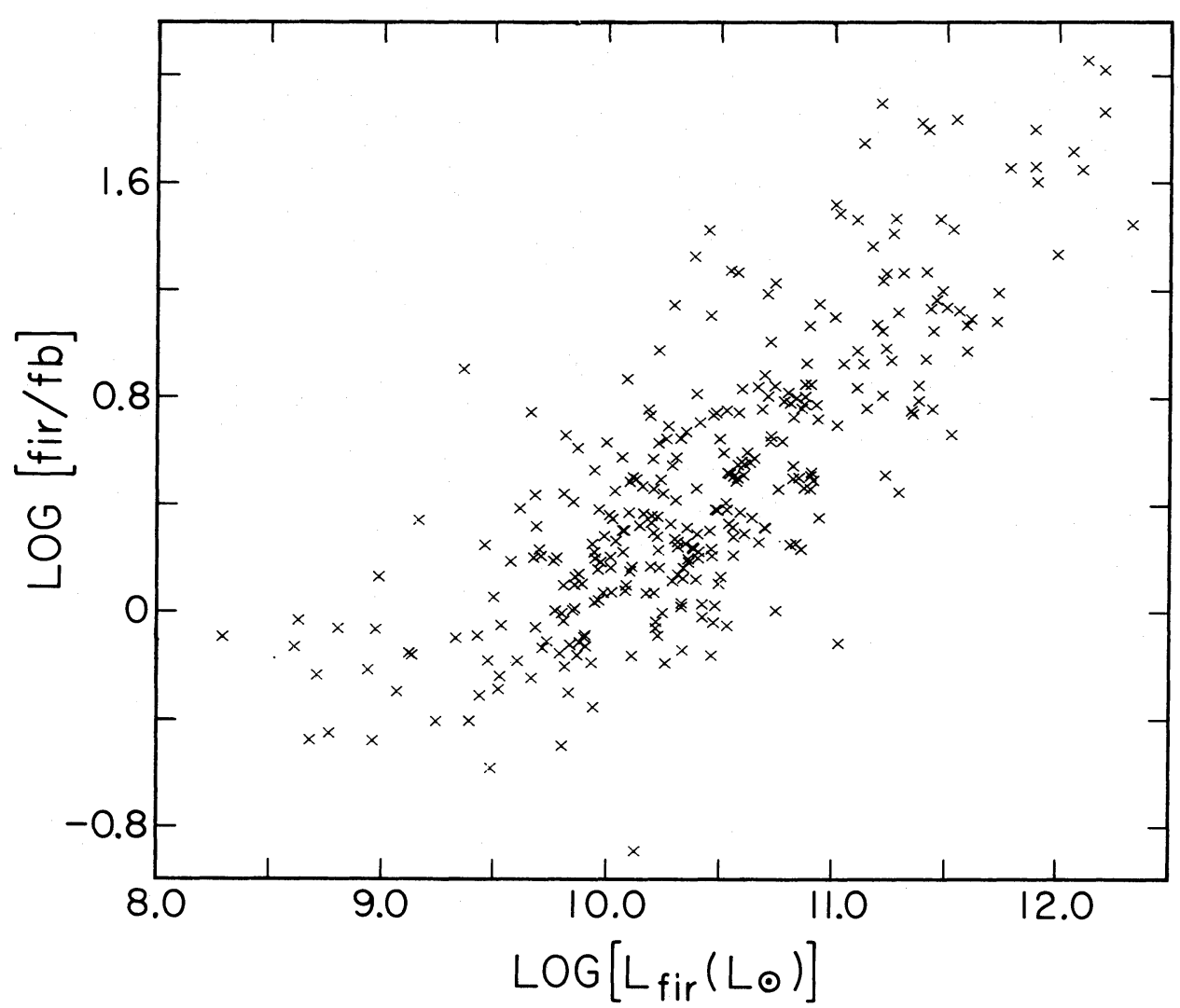

Fig. $5 a$

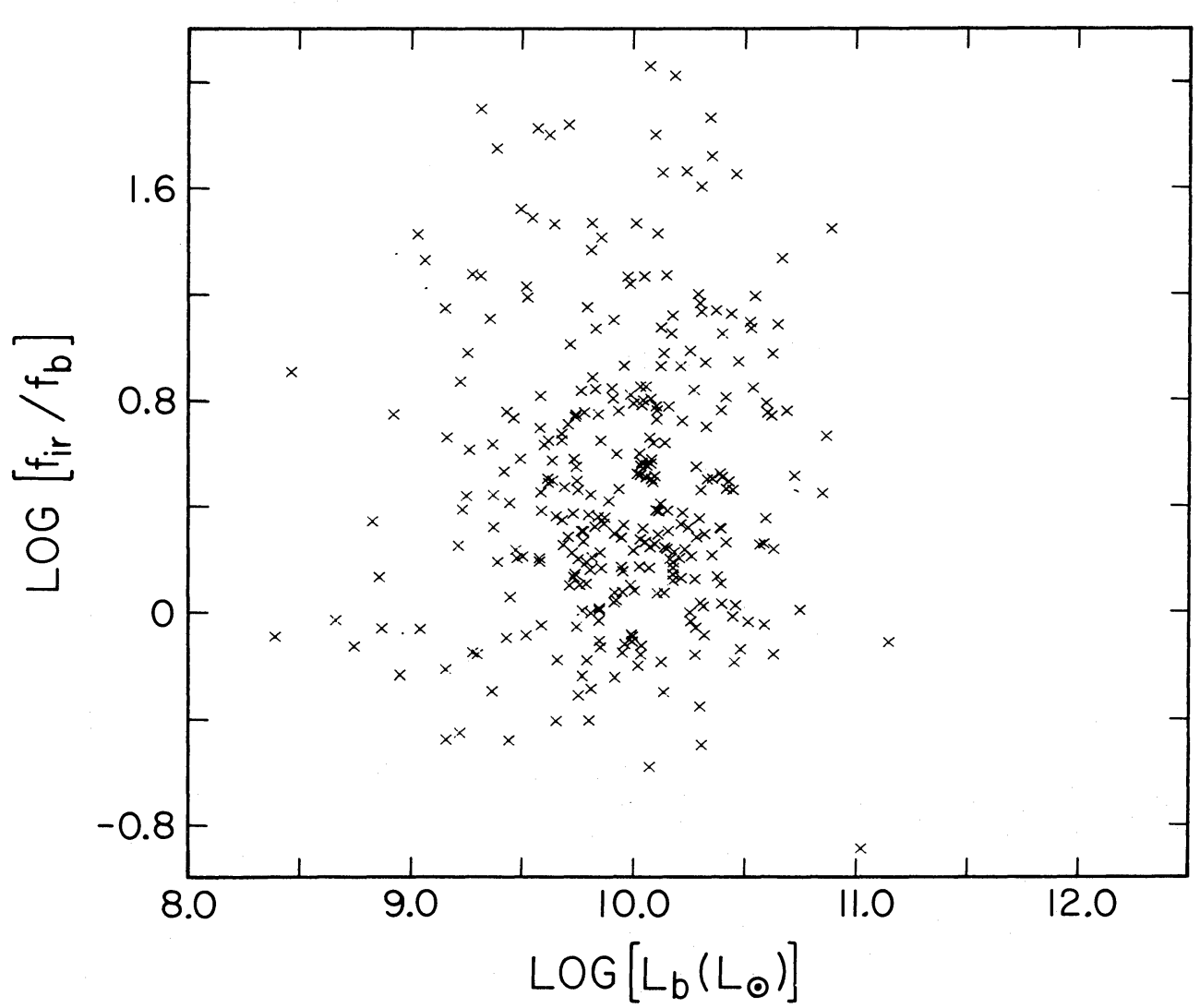

FIG. $5 b$

FIG. 5.- (a) Plot of ratio of far-infrared to blue flux vs. far-infrared luminosity for bright galaxy sample. Increase in average ratio of far-infrared to blue flux is closely linearly proportional to far-infrared luminosity. (b) Plot of ratio of far-infrared to blue flux vs. blue luminosity for bright galaxy sample. There is no apparent correlation between these quantities. 


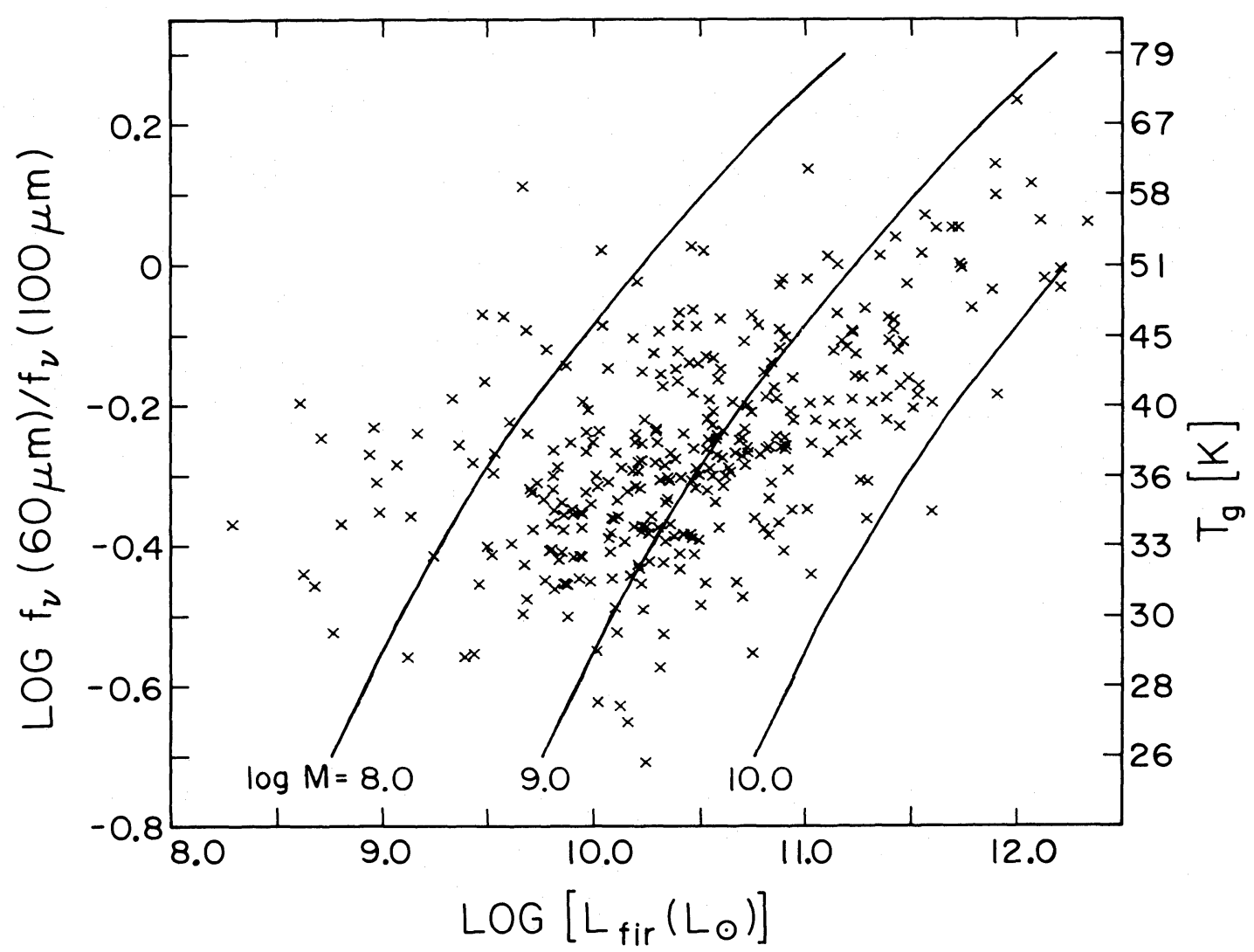

FIG. $6 a$

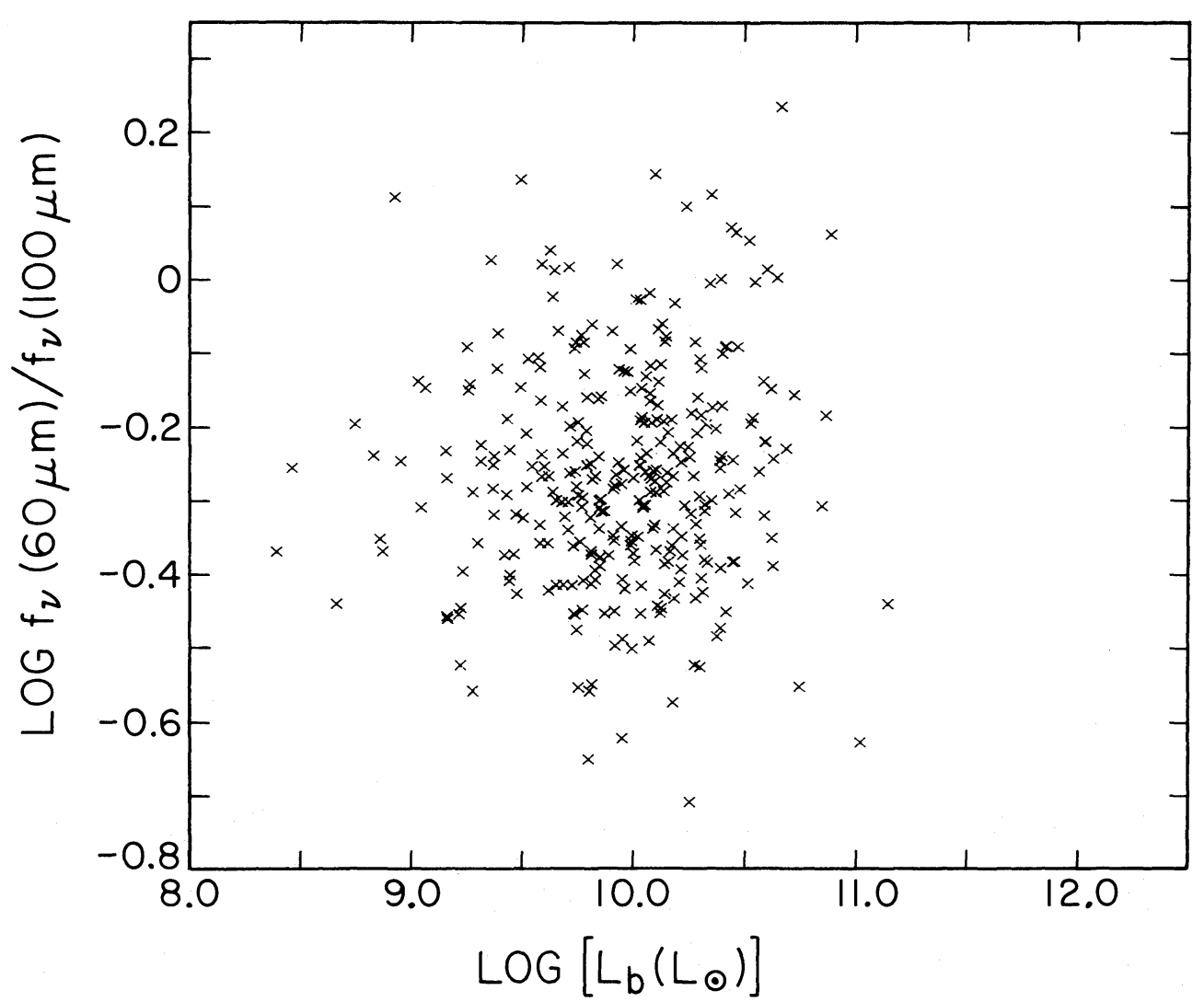

FIG. $6 b$

Fig. 6.-(a) Plot of ratio of $60 \mu \mathrm{m} / 100 \mu \mathrm{m}$ flux densities vs. far-infrared luminosity bright galaxy sample. Ordinate is also shown as grain temperature for grains having emissivity $\epsilon \propto v$. Lines of gas mass of $10^{8}, 10^{9}$, and $10^{10} M_{\odot}$ are drawn, where $M_{\rho} / M_{d}=200$ has been assumed. Dust is assumed to radiate with temperature given by right hand temperature scale, and $100 \mu \mathrm{m}$ dust opacity is taken from Draine and Lee (1984). (b) Plot of ratio of $60 \mu \mathrm{m} / 100 \mu \mathrm{m}$ flux densities vs. blue luminosity for bright galaxy sample. No correlation is apparent between these quantities. 


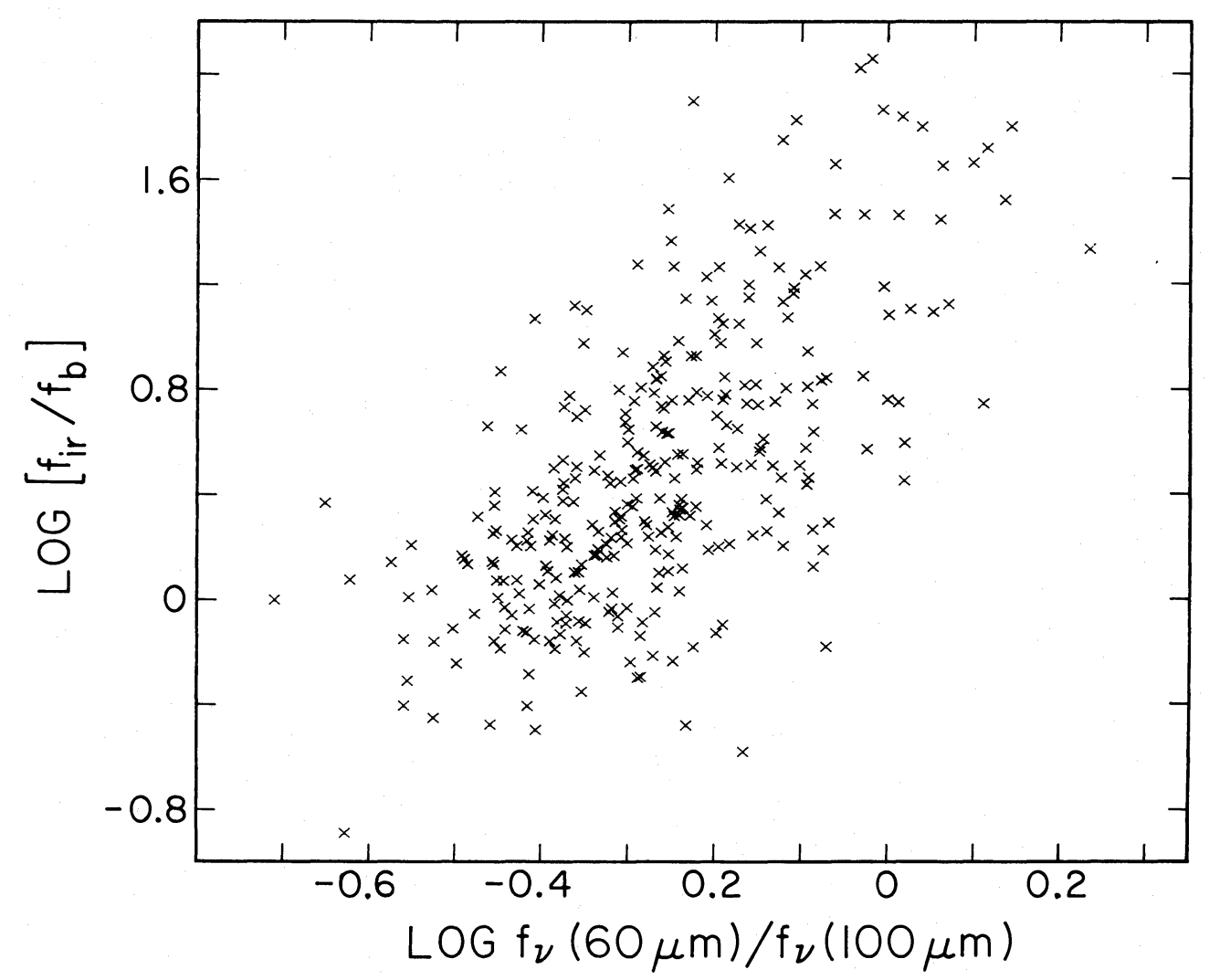

Fig. 7.-Plot of ratio of far-infrared to blue flux vs. $60 \mu \mathrm{m} / 100 \mu \mathrm{m}$ flux density for bright galaxy sample. There is a tendency for higher values of $f_{\text {FIR }} / f_{b}$ to be associated with higher $60 \mu \mathrm{m} / 100 \mu \mathrm{m}$ ratios.

and

$$
\sigma_{\rho}=\left(\frac{4 \pi}{\Omega}\right)\left(\sum \frac{1}{V_{m}^{2}}\right)^{1 / 2}
$$

where $\Omega$ is the solid angle of the survey, and $V_{m}$ is the maximum volume to which the object could have been detected in the survey, and the summation is over all galaxies in a given luminosity bin (Schmidt 1968).

The more sophisticated estimator of Felton (1976) reduces to the above expression for a uniform flux limit for the survey, as is the case here. Here $V_{m}$ was individually estimated for each galaxy in the sample. The $K$ correction, determined using a power-law slope (Sandage 1975) defined by the observed $60 \mu \mathrm{m}$ and $100 \mu \mathrm{m}$ flux densities, was taken into account in calculating $V_{m}$. Since all redshifts are comparatively small, this power law defines the spectrum near $60 \mu \mathrm{m}$ better than the slope between $25 \mu \mathrm{m}$ and $60 \mu \mathrm{m}$ does.

The space densities as a function of luminosity are given in Table 2, along with the number of galaxies in each luminosity bin, the uncertainty in the space density, and the average $V / V_{m}$ for that bin along with its uncertainty. The quantities are given both including and excluding galaxies deemed to be associated with the Virgo cluster. The luminosity function that excludes

TABLE 2

LUMINOSITY FUNCTION AT 60 MicRONS

\begin{tabular}{|c|c|c|c|c|c|c|}
\hline \multirow[b]{2}{*}{$\begin{array}{c}\log L^{\mathbf{a}} \\
\left(L_{\odot}\right)\end{array}$} & \multicolumn{3}{|c|}{ All Galaxies } & \multicolumn{3}{|c|}{ GALAXIES: Virgo EXClUdED } \\
\hline & $N$ & $\left(\mathrm{Mpc}^{-3} \mathrm{mag}^{-1}\right)$ & $V / V_{m}$ & $N$ & $\left(\mathrm{Mpc}^{-3} \mathrm{mag}^{-1}\right)$ & $V / V_{m}$ \\
\hline 8.2. & 4 & $3.2 \pm 1.7 \times 10^{-2}$ & $0.50 \pm 0.13$ & $\ldots$ & $\cdots$ & $\cdots$ \\
\hline $8.6 \ldots$ & 8 & $1.6 \pm 0.6 \times 10^{-2}$ & $0.36 \pm 0.09$ & $\ldots$ & & $\ldots$ \\
\hline $9.0 \ldots$ & 11 & $5.6 \pm 1.8 \times 10^{-3}$ & $0.45 \pm 0.08$ & 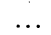 & 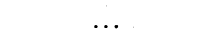 & . \\
\hline $9.4 \ldots \ldots \ldots$ & 41 & $4.4 \pm 0.7 \times 10^{-3}$ & $0.59 \pm 0.05$ & 23 & $2.6 \pm 0.6 \times 10^{-3}$ & $0.55 \pm 0.07$ \\
\hline $9.8 \ldots \ldots \ldots$ & 62 & $2.1 \pm 0.3 \times 10^{-3}$ & $0.44 \pm 0.03$ & 52 & $1.8 \pm 0.3 \times 10^{-3}$ & $0.47 \pm 0.04$ \\
\hline $10.2 \ldots$ & 78 & $6.6 \pm 0.8 \times 10^{-4}$ & $0.42 \pm 0.03$ & 75 & $6.3 \pm 0.8 \times 10^{-4}$ & $0.43 \pm 0.03$ \\
\hline $10.6 \ldots \ldots \ldots$ & 53 & $1.1 \pm 0.2 \times 10^{-4}$ & $0.45 \pm 0.04$ & $\ldots$ & $\ldots$ & $\ldots$ \\
\hline $11.0 \ldots \ldots \ldots$ & 29 & $1.5 \pm 0.3 \times 10^{-5}$ & $0.58 \pm 0.05$ & $\ldots$ & $\ldots$ & $\ldots$ \\
\hline $11.4 \ldots \ldots \ldots$ & 25 & $4.0 \pm 0.8 \times 10^{-6}$ & $0.47 \pm 0.06$ & $\ldots$ & $\ldots$ & $\ldots$ \\
\hline $11.8 \ldots \ldots \ldots$ & 9 & $3.1 \pm 1.1 \times 10^{-7}$ & $0.46 \pm 0.09$ & $\ldots$ & $\ldots$ & $\ldots$ \\
\hline $12.2 \ldots \ldots \ldots$ & 4 & $4.3 \pm 2.2 \times 10^{-8}$ & $0.29 \pm 0.15$ & $\ldots$ & $\ldots$ & $\ldots$ \\
\hline
\end{tabular}

${ }^{\mathrm{a}} L(60 \mu \mathrm{m})=v L_{v}(60 \mu \mathrm{m})$. 
the Virgo cluster takes no account of the volume excluded from the sample; this volume is less than $8 \%$ of the total surveyed volume in all bins.

In Figure 8 the quantity $V / V_{m}$ as a function of $60 \mu \mathrm{m}$ luminosity is plotted. Where appropriate, the $V / V_{m}$ data with the Virgo galaxies included and excluded are shown. There are no significant deviations from the value of 0.5 expected for a sample that homogeneously fills the volume, with the maximum deviation from the uniform case being for the bin $\log \left[v L_{v}(60 \mu \mathrm{m})\right]=10.2$ with $V / V_{m}$ of 0.43 , a $2.5 \sigma$ result. For the entire sample, $V / V_{m}=0.47 \pm 0.02$, again not significantly different from 0.5 . The effect of the Virgo cluster can also be seen in the points where these galaxies are included. In the bins at $\log \left[v L_{v}(60 \mu \mathrm{m})\right]=9.4,9.8$, and 10.2 the inclusion of the Virgo cluster galaxies makes $V / V_{m}$ differ significantly from 0.5 .

Other $60 \mu \mathrm{m}$ luminosity functions have been derived based on different samples taken from the IRAS data (e.g., Lawrence et al. 1986; Rieke and Lebofsky 1986; Smith et al. 1987). All of these $60 \mu \mathrm{m}$ luminosity functions are compared with the luminosity function for the bright galaxy sample in Figure 9. All the luminosity functions have been converted to the units adopted here. In the case of the Lawrence et al. results, the only conversion necessary was for different Hubble constants. No attempt was made to account for differing value of $q_{0}$, since the largest redshifts in the bright galaxy sample are less than $z=0.1$. For the Smith et al. results the only conversions necessary were for the Hubble constant and a different multiplier of $L_{v}(60 \mu \mathrm{m})$. For the Rieke and Lebofsky sample, the relation $L=1.6 \times v L_{v}(60 \mu \mathrm{m})$ was adopted based on the distribution of flux ratios presented in their work. As can be seen from Figure 9, the agreement between the luminosity functions derived from different samples is excellent.

While the criteria used to define the bright galaxy sample were based on $60 \mu \mathrm{m}$ flux density and optical identification, it is interesting to consider whether this sample differs from galaxy samples chosen based on infrared color criteria for studies of the spatial distribution of infrared galaxies (e.g., Yahil, Walker, and Rowan-Robinson 1986; Meiksin and Davis 1986). All of the objects in the bright galaxy sample meet the criteria for inclusion in both of these samples, while neither Yahil, Walker, and Rowan-Robinson nor the Meiksin and Davis samples include objects that would be excluded based on color criteria from the bright galaxy sample. Thus it appears that there are no substantial differences between these samples, and the bright galaxy sample can be used as a fiducial point for fainter, more distant samples of infrared selected galaxies.

Rieke and Lebofsky (1986) have shown that the Schechter (1976) type luminosity function falls below the observed luminosity function at high luminosity. This is also seen in Figure 9. Two power laws fitted to the observed $60 \mu \mathrm{m}$ luminosity function are also shown in Figure 9. At low luminosities the best-fit power law gives a slope $\rho \sim L^{-0.8}$, while at high luminosities the best-fit slope is $\rho \sim L^{-2.0}$, again in good agreement with the best-fit power law slope of -2.1 estimated by Rieke and Lebofsky. The slope at low luminosity agrees well with the slope for this region of -0.8 derived by Lawrence et al. (1986), while at the high-luminosity end the slope is steeper than that

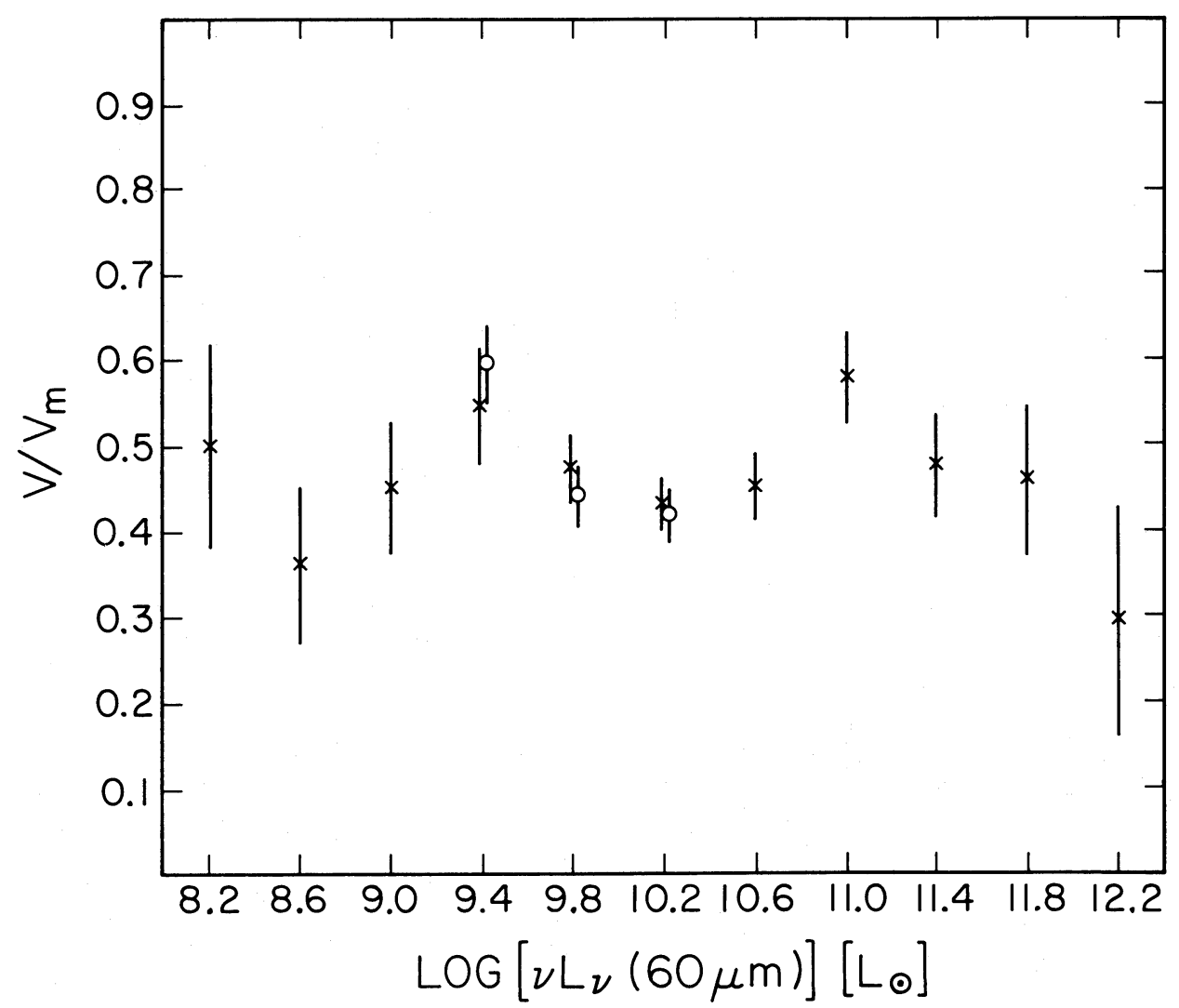

FIG. 8.-Mean $V / V_{m}$ for the galaxies in bright galaxy sample plotted vs. luminosity of appropriate bin. Crosses represent bins where Virgo cluster galaxies have been excluded, open circles represent all galaxies in those luminosity bins. Only with inclusion of Virgo galaxies are any statistically significant deviations from value of 0.5 expected for galaxies uniformly distributed in volume. 


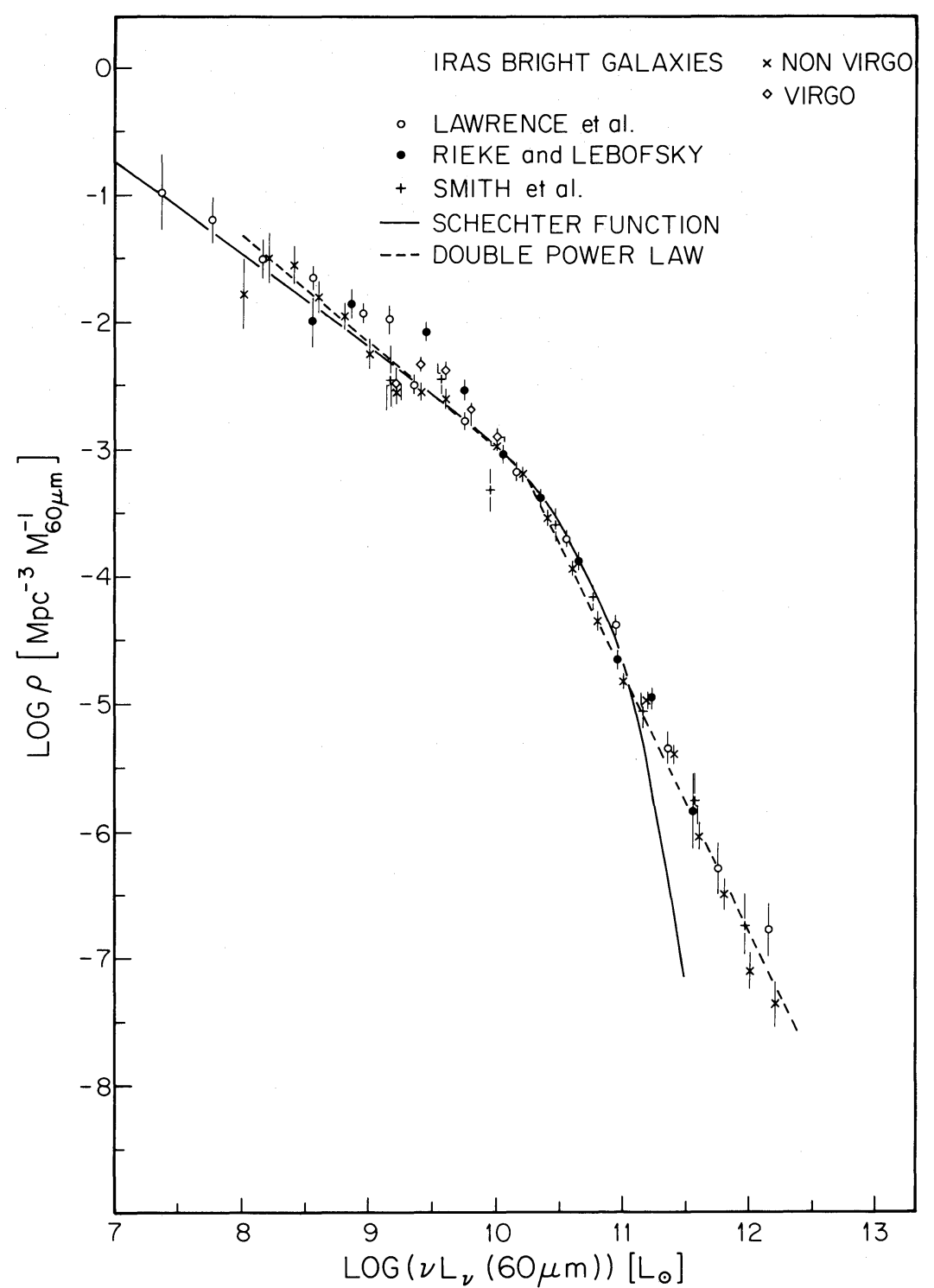

FIG. 9.-The $60 \mu \mathrm{m}$ luminosity functions from different samples, compared to that derived here for bright galaxy sample. Luminosity functions have been adjusted to same Hubble constant $\left(H_{0}=75 \mathrm{~km} \mathrm{~s}^{-1} \mathrm{Mpc}^{-1}\right)$, same definition of luminosity $\left(v L_{v}[60 \mu \mathrm{m}]\right)$, and bins represent space density in galaxies per $\mathrm{Mpc}^{3}$ per magnitude interval in luminosity. Otherwise no adjustments have been made to different luminosity functions. Bright galaxy luminosity function is shown excluding Virgo (crosses), and three bins, including Virgo galaxies (open diamonds). Other luminosity functions shown are from Lawrence et al. 1986 (open circles), Rieke and Lebofsky 1986 ( filled circles), and Smith et al. 1987 (plus signs). Solid line represents a "by eye" fit of a Schechter function to all luminosity functions. Note substantial discrepancy at high luminosity end of luminosity function. Dashed line represents best fit of two power laws to bright galaxy luminosity function.

of -1.7 from Lawrence et al., consistent with the observed space densities in the sample of Lawrence et al. being higher at the highest luminosities. The luminosity of the break between the two power laws is $1.7 \times 10^{10} L_{\odot}$; this is the most frequent luminosity seen in this flux-limited sample.

Whether the $60 \mu \mathrm{m}$ luminosity function can be extrapolated to higher luminosities is quite uncertain. Based on the luminosity function derived above, approximately three objects should have been discovered in the bright galaxy sample with luminosities placing them in the next greater luminosity bin. Clearly the absence of any such examples has no statistical significance, while a handful of fainter, more luminous objects are already known to exist in the IRAS survey (e.g., 3C 48 and Mrk 1014; Neugebauer, Soifer, and Miley 1985). Furthermore, two infrared "loud" objects have been found in the luminosity range $10^{13} L_{\odot}$ (Kleinmann and Keel 1987; Vader 1986). The existence of these objects suggests that an extrapolation of the observed luminosity function by an order of magnitude is not unrealistic. However, the extension of the far-infrared luminosity function to such luminosities must await a survey of sufficient numbers of IRAS galaxies.

It is tempting to use the different luminosity functions to search for potential evolutionary effects. The largest range in distance is achieved by comparing the bright galaxy luminosity function with that of Lawrence et al. (1986), where the completeness limit was $0.85 \mathrm{Jy}$, and by selecting the highest possible luminosity bin for comparison. Figure 9 shows a suggestion of the luminosity function changing in the expected way if there were an increased density of high-luminosity infrared galaxies in the past. Formally, the increase in density of 


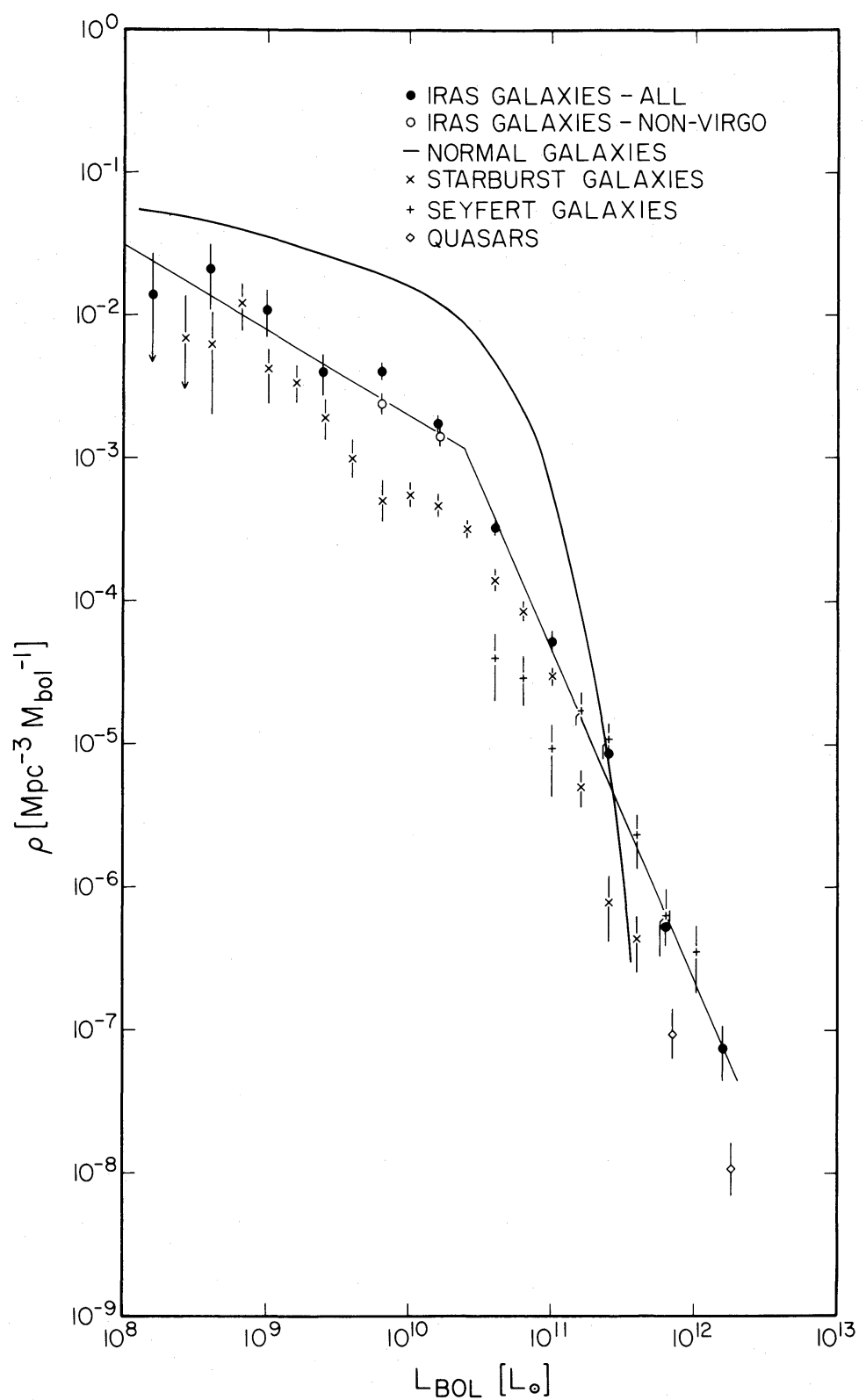

Fig. 10.-Luminosity functions of a variety of classes of extraglactic sources, normalized to same Hubble constant $\left(H_{0}=75 \mathrm{~km} \mathrm{~s}^{-1} \mathrm{Mpc}^{-1}\right)$ and plotted in units of bolometric luminosity. Filled and open circles represent far-infrared luminosity function derived for bright galaxy sample, including and excluding the Virgo cluster respectively. Solid curve represents analytical fit to normal galaxy luminosity function taken from Schechter (1976) that agrees with many observed luminosity functions (Felton, 1977); crosses represent the optically selected starburst galaxies, and plus signs represent the optically selected Seyfert galaxies, both taken from Huchra (1977). Open diamonds represent optically selected quasars taken from Schmidt and Green (1983). Corrections applied to convert from blue luminosity to bolometric luminosity are described in appendix. Straight lines represent best fit of two power laws to bright galaxy luminosity function excluding Virgo galaxies.

galaxies in the range $\log \left[v L_{v}(60 \mu \mathrm{m})\right]=12.0-12.4$ is a factor of $\sim 3$. This highly uncertain increase in space density with redshift is consistent with the analysis of the counts of $60 \mu \mathrm{m}$ sources at the $50 \mathrm{mJy}$ level by Hacking, Condon, and Houck (1987).

\section{b) Comparison with Other Classes of Objects}

One goal of the present study is to understand the significance of far-infrared emission in the local universe. This requires comparing the luminosities emitted at different wavelengths by very different classes of objects. In Figure 10, the bolometric luminosity functions of a variety of different classes of extragalactic objects are plotted. The far-infrared luminosity described above has been adopted as the bolometric luminosity for the IRAS bright galaxy sample. The total far-infrared luminosities calculated in this way are $\sim 50 \%$ greater than the $60 \mu \mathrm{m}$ luminosities. This ignores an additional contribution of $\sim 25 \%$ to the total luminosity from the emission at shorter wavelengths.

Table 3 gives the far-infrared luminosity function. The calculations were done as for the $60 \mu \mathrm{m}$ space densities; the only difference was the binning by total far-infrared luminosity, 
TABLE 3

FAR-INFRARED LUMINOSITY FUNCTION

\begin{tabular}{|c|c|c|c|c|}
\hline \multirow[b]{2}{*}{$\underset{\left(L_{\odot}\right)}{\log L_{\mathrm{FIR}}{ }^{\mathrm{a}}}$} & \multicolumn{2}{|c|}{ All Galaxies } & \multicolumn{2}{|c|}{ GalaXIES: VIRGO EXCLUDED } \\
\hline & $N$ & $\left(\mathrm{Mpc}^{-3} \mathrm{mag}^{-1}\right)$ & $N$ & $\left(\mathrm{Mpc}^{-3} \mathrm{mag}^{-1}\right)$ \\
\hline $8.2 .$. & 1 & $1.5 \pm 1.5 \times 10^{-2}$ & $\ldots$ & $\ldots$ \\
\hline $8.6 \ldots$ & 5 & $2.3 \pm 1.1 \times 10^{-2}$ & $\ldots$ & $\ldots$ \\
\hline $9.0 \ldots$ & 9 & $1.2 \pm 0.4 \times 10^{-2}$ & $\ldots$ & $\ldots$ \\
\hline $9.4 \ldots \ldots \ldots$ & 14 & $4.3 \pm 1.3 \times 10^{-3}$ & $\ldots$ & $\ldots$ \\
\hline $9.8 \ldots \ldots \ldots$ & 52 & $4.2 \pm 0.6 \times 10^{-3}$ & 31 & $2.3 \pm 0.5 \times 10^{-3}$ \\
\hline $10.2 \ldots \ldots \ldots$ & 79 & $1.8 \pm 0.3 \times 10^{-3}$ & 70 & $1.5 \pm 0.2 \times 10^{-3}$ \\
\hline $10.6 \ldots \ldots \ldots$ & 68 & $3.5 \pm 0.5 \times 10^{-4}$ & 67 & $3.4 \pm 0.5 \times 10^{-4}$ \\
\hline $11.0 \ldots$ & 44 & $5.6 \pm 0.9 \times 10^{-5}$ & $\ldots$ & $\ldots$ \\
\hline $11.4 \ldots \ldots \ldots$ & 35 & $9.2 \pm 1.7 \times 10^{-6}$ & $\ldots$ & $\ldots$ \\
\hline $11.8 \ldots \ldots \ldots$ & 11 & $5.7 \pm 1.8 \times 10^{-7}$ & $\ldots$ & $\ldots$ \\
\hline $12.2 \ldots \ldots \ldots$ & 6 & $7.9 \pm 3.4 \times 10^{-8}$ & $\ldots$ & $\ldots$ \\
\hline
\end{tabular}

${ }^{\text {a }} L_{\text {FIR }}$ is defined in the text.

rather than $60 \mu \mathrm{m}$ luminosities. The data in Table 3 are used below to compare "bolometric luminosity functions" of different luminosity components in galaxies.

The bright galaxy sample is only strictly complete at $60 \mu \mathrm{m}$, and not in a bolometric sense over the entire far-infrared wavelength range. Thus there could be cold or warm objects that are extremely numerous but would not be included in the bright galaxy sample. The PSC was searched using $60 \mu \mathrm{m} /$ $100 \mu \mathrm{m}$ and $25 \mu \mathrm{m} / 60 \mu \mathrm{m}$ color criteria intended to determine if objects colder or warmer than those selected here might be numerous compared to the bright galaxy sample. While both cold and warm galaxies were found, based on the additional searches of the PSC such objects are likely to comprise less than $25 \%$ of the galaxies at a given far-infrared luminosity within a given volume. We conclude that the bright galaxy sample represents a legitimate sample of the local universe in the far-infrared.

For comparison, luminosity functions taken from the literature for " normal galaxies," "starburst galaxies," Seyfert galaxies, and quasars are included in Figure 10. The published luminosity functions are given in terms of $M_{b}$, i.e., absolute blue luminosity, so it was necessary to estimate a bolometric correction for each of the classes of objects. The steps taken to derive these bolometric corrections were described in Paper I, but the details are repeated in the Appendix for completeness.

It is important to remember, when comparing the different luminosity functions, that some galaxies can simultaneously be classified in more than one category of object, and are not necessarily evaluated at the same luminosity in each category. Figure 10 should thus be viewed as a comparison of the space density of sources of far-infrared luminosity with that of sources of luminosity that emerge predominantly at shorter wavelengths. For example, the starburst galaxies comprise a subset of the "normal" galaxies, and their luminosity is estimated in a similar way. The bolometric corrections described in the Appendix for these classes of galaxies do not include the far-infrared luminosity emitted by such galaxies (above that in the stellar photospheres). For the Seyferts and quasars, an estimate of the far-infrared luminosity has been included in the calculation of the luminosity, but this is only $10 \%-15 \%$ of the total luminosity of these objects.

One can immediately see from Figure 10 that the emission from infrared bright galaxies represents a significant component of luminosity in the local universe. The infrared gal- axies are more numerous by a factor of $\sim 3$ than Markarian starburst galaxies at $L_{\text {FIR }} \lesssim 10^{10} L_{\odot}$. In the range $10^{10}$ $L_{\odot}-10^{11} L_{\odot}$, the densities of the two classes of objects are comparable. For luminosities above $\sim 2 \times 10^{11} L_{\odot}$ infrared luminous galaxies appear to be the dominant source of luminosity in the local universe, having virtually the same space densities as the Seyferts at the lower end of this range, and a significantly greater space density than quasars at the higher luminosities. A detailed discussion of the spectroscopic and morphological properties of the galaxies in this highest range of infrared luminosities of the bright galaxy sample is in preparation (Sanders et al. 1987a).

For luminosities below $\sim 2 \times 10^{11} L_{\odot}$, normal galaxies dominate the space densities in the local universe. From the far-infrared luminosity function the contribution to the luminosity density of the local universe can be estimated. The infrared galaxies with far-infrared luminosities greater than $10^{8} L_{\odot}$ produce $\sim 9 \times 10^{7} L_{\odot} \mathrm{Mpc}^{-3}$ in far-infrared emission, with $4 \times 10^{7} \mathrm{~L}_{\odot} \mathrm{Mpc}^{-3}$ being generated in galaxies with farinfrared luminosities greater than $10^{10} L_{\odot}$. By comparison, the normal galaxies produce a bolometric luminosity density of $\sim 4 \times 10^{8} \mathrm{~L}_{\odot} \mathrm{Mpc}^{-3}$, where the integrated blue luminosity density taken from Felton (1977) and Yahil, Sandage, and Tammann (1980), corrected to $H_{0}=75 \mathrm{~km} \mathrm{~s}^{-1} \mathrm{Mpc}^{-1}$, has been corrected by the same bolometric correction as adopted for normal galaxies. Thus the far-infrared luminosity is $\sim 25 \%$ of the stellar luminosity of galaxies. Felton and Yahil, Sandage, and Tammann estimate the luminosity density of normal galxies by reducing the absolute scale factor for the local luminosity function to that determined from the number counts at faint magnitudes (see Appendix). There is no evidence that this same factor should be applied to the infrared luminosity function. Indeed, the agreement betwen the luminosity functions of the bright galaxy sample and that of Lawrence et al. (1986) suggests that the $60 \mu \mathrm{m}$ luminosity function remains constant in normalization when the median galaxy distance changes from $\sim 30 \mathrm{Mpc}$ to $\sim 100 \mathrm{Mpc}$. Perhaps the absence of elliptical and dwarf galaxies from the infrared selected samples contributes to the more uniform density.

At luminosities greater than $\sim 10^{10} L_{\odot}$ it is likely that star formation is the dominant form of energy generation in infrared bright galaxies (Becklin 1987), at least until the very highest luminosities. Several authors (Persson and Helou 1986; Helou $1986 b$; Rowan-Robinson and Crawford 1987; de Jong and Brink 1987) have suggested that a significant fraction of the far-infrared luminosity in less active galaxies is recycled stellar radiation not directly associated with star-formation regions. Thus, directly or indirectly, star formation accounts for between $60 \%$ and $80 \%$ of the far-infrared luminosity generated in the local universe.

The total space density of galaxies with far-infrared luminosities greater than $10^{11} L_{\odot}$ is $\sim 1.2 \times 10^{-5} \mathrm{Mpc}^{-3}$. Figure $5 a$ shows that $85 \%$ of these galaxies have blue luminosities greater than $10^{10} L_{\odot}$. From Christensen (1975), the space density of normal galaxies with $L_{0}>10^{10} L_{\odot}$ is $3.4 \times 10^{-3}$ $\mathrm{Mpc}^{-3}$, or $\sim 0.3 \%$ of the galaxies with $L_{b}>10^{10} L_{\odot}$ have $L_{\text {FIR }}>10^{11} L_{\odot}$. If the infrared bright phase has a lifetime $t_{\text {IR }}$ and the optical phase has a lifetime $t_{b}$ then the fraction of galaxies that have undergone such an infrared active phase is $0.003 \times t_{b} / t_{\mathrm{IR}}$. If the overall normalization of the optical luminosity function is reduced by a factor of 2.3 (Felton 1977) while the far-infrared luminosity function remains constant, as suggested in Figure 9, then the fraction of galaxies undergoing this 
phase become $0.007 \times t_{b} / t_{\mathrm{IR}}$. As noted in Paper $\mathrm{I}$, if $t_{b} \sim 10^{10}$ $\mathrm{yr}$ and the infrared bright phase is a nonrecurring starburst phase with $t_{\mathrm{IR}}<10^{8} \mathrm{yr}$ (Rieke et al. 1980; Gerhz, Sramek, and Weedman 1983), then a significant fraction, perhaps more than $50 \%$, of galaxies with $L_{b}>10^{10} L_{\odot}$ must have undergone such an infrared active period. If $t_{b}$ is as small as $10^{9} \mathrm{yr}$ and $t_{\mathrm{IR}}$ is $\sim 10^{8} \mathrm{yr}$, then almost $10 \%$ of such galaxies could undergo such a phase.

\section{SUMMARY}

From a complete sample of the brightest galaxies detected at $60 \mu \mathrm{m}$ in the IRAS all-sky survey, we have found the following:

1. Far-infrared emission is a significant luminosity component in the local universe, representing $25 \%$ of the luminosity emitted by stars in the same volume. Above $10^{11} L_{\odot}$ the infrared luminous galaxies are the dominant population of objects in the universe, being as numerous as the Seyfert galaxies, and more numerous than quasars at higher luminosities.

2. The infrared luminosity appears to be independent of the optical luminosity of galaxies. Most infrared bright galaxies appear to require much, if not all, of their interstellar matter to be contributing to the observed infrared luminosity.

3. Approximately $60 \%-80 \%$ of the far-infrared luminosity of the local universe can be attributed, directly or indirectly, to recent or ongoing star formation.

It is a pleasure to thank members of the IPAC staff for assistance in assembling IRAS data, our night assistants at Palomar, Juan Carasco and Skip Staples, for assistance in obtaining the optical spectra and optical photometry, and George Helou, Paul Schechter, and Jeremy Mould for illuminating conversations. This research was supported in part by NASA through the IRAS Extended Mission program, and in part by the NSF. G. E. D. is supported by NASA contract NAS5-25451. B. F. M. is supported in part by the Natural Sciences and Engineering Research Council of Canada and by the Canada Council through a Killam Fellowship. This is contribution No. 4427 of the Division of Geological and Planetary Sciences.

\section{APPENDIX}

\section{CONVERSION OF LUMINOSITY FUNCTIONS TO BOLOMETRIC LUMINOSITIES}

Nearly all of the luminosity functions derived for classes of extragalactic objects are given in units of $M_{B}$. Since the comparison of the far-infrared luminous galaxies with other classes of extragalactic objects requires measuring in comparable units of luminosity, and blue luminosity is not applicable to the infrared luminous galaxies, bolometric luminosity has been selected for comparison of the various luminosity functions.

Felton (1977) has discussed nine optical luminosity functions derived for nearby galaxies, and has concluded that all but one agree. The analytic form of this function formulated by Schechter (1976) is a good fit to these data, and is adopted here as the optical luminosity function of the normal galaxies. Felton has suggested that the local luminosity function for normal galaxies is too high by a factor of $\sim 2.3$ when comparing the number counts of galaxies at fainter magnitudes with those predicted from the local luminosity function. Since the IRAS bright galaxy luminosity function has been derived over roughly the same distances as the normal galaxy luminosity function, no adjustment has been made in the normalization of the normal galaxy luminosity function. An average $B-V$ color for the normal galaxies was taken as $0.8 \mathrm{mag}$, and a bolometric correction of 0.9 mag was adopted. This bolometric correction is consistent with the $V-K$ colors of typical galaxies (Aaronson 1977; Johnson 1966).

The non-Seyfert Markarian galaxies represent the most complete sample of optically selected starburst galaxies (Bohuski, Fairall, and Weedman 1978) and the luminosity function for these galaxies was taken from the work of Huchra (1977). A mean $B-V$ color of $0.5 \mathrm{mag}$ and a bolometric correction of $1.2 \mathrm{mag}$ are adopted for these galaxies (Huchra 1977; Balzano 1983). This correction includes contributions for the photospheres of late-type and hot stars in these galaxies.

The luminosity function for Seyfert galaxies, assumed to be characterized by the luminosity function for the Markarian Seyferts, was taken from Huchra (1977), while the luminosity function for the quasars was taken from Schmidt and Green (1983). The bolometric correction for both of these classes of objects was assumed to be the same and was estimated as $9 \times v L_{v}(0.43 \mu \mathrm{m})$. This was derived by assuming a three-step power-law flux distribution, where the slope $\left(f_{v} \approx v^{\alpha}\right)$ was taken as -1 for $3 \times 10^{12}-$ $3 \times 10^{14} \mathrm{~Hz},-0.5$ for $3 \times 10^{14}-3 \times 10^{15} \mathrm{~Hz}$, and -1.5 for $3 \times 10^{15}-3 \times 10^{16} \mathrm{~Hz}$ (Malkan and Sargent 1982; Malkan 1983; Elvis et al. 1986; O'Dell, Scott, and Stein 1986). A comparison of this approximation with the integrated energy distributions of a variety of AGNS from 0.1 to $100 \mu \mathrm{m}$ (Edelson and Malkan 1986) indicates that it represents the total bolometric luminosity of these objects to within $30 \%$.

Aaronson, M. 1977, Ph.D. thesis, Harvard University.

Aaronson, M. et al. 1982a, Ap. J.Suppl., 50, 241.

Aaronson, M., Huchra, J., Mould, J., Schechter, P. L., and Tully, R. B. 1982b, Ap. J., 258, 64.

Aaronson, M., and Mould, J. 1983, Ap. J., 265, 1.

Allen, C. W. 1973, Astrophysical Quantities 3d ed.;(London: Athlone).

Balzano, V. 1983, Ap. J., 268, 602 .

Becklin, E. E. 1987, in Star Formation Galaxies, ed. C. J. Persson (Washington, DC: US Government Printing Office).

Bohuski, P. J., Fairall, A. P., and Weedman, D. W. 1978, Ap. J., 221, 776

Bothun, G., Lonsdale, C. J., and Rice, W. L. 1987, in preparation.

Cataloged Galaxies and Quasars Observed in the IRAS Survey. 1985, prepared

by C. J. Lonsdale, G. Helou, J. C. Good, and W. L. Rice (Pasadena: Jet

Propulsion Laboratory D-1932).

\section{REFERENCES}

Chester, T. J. 1986, in Light on Dark Matter, ed. F. Israel (Dordrecht: Reidel), p. 3.

Christensen, C. G. 1975, A.J., 80, 282.

de Jong, T, and Brink, K. 1987, in Star Formation in Galaxies, ed. C. J. Persson (Washington, DC: US Government Printing Office), in press.

de Jong., et al. 1984, Ap. J. (Letters), 278, L67.

de Vaucouleurs, G., de Vaucouleurs, A., and Corwin, H. 1976, Second Reference Catalogue of Bright Galaxies (Austin: University of Texas Press).

Draine, B. T., and Lee, H. M. 1984, Ap. J., 258, 111.

Edelson, R., and Malkan, M. 1986, Ap.J., 308, 59.

Elvis, M., Green, R. F., Bechtold, J., Schmidt, M., Neugebauer, G., Soifer, B. T., Matthews, K., and Fabbiano, G. 1986, Ap. J., 310, 291.

Felten, J. E. 1976, Ap. J., 207, 700.

. 1977, A.J., 82, 861. 
Gallagher, J. S., and Hunter, D. A. 1984, Ann. Rev. Astr. Ap., 22, 37

Gehrz, R. D., Sramek, R. A., and Weedman, D. W. 1983, Ap. J., 267, 551.

Hacking, P., Condon, J., and Houck, J. R. 1987, Ap. J., submitted.

Helou, G. 1986a, in Workshop on Star-Forming Dwarf Galaxies, ed. D. Knuth T. X. Thuan, and J. Trans Thanh Van, (Gif sur Yvette: Editions Frontiers), p. 1.

. 1986b, Ap.J. (Letters), 311, L33.

Huchra, J. 1977, Ap. J.Suppl., 35, 171.

Huchra, J., Davis, M., Latham, D., and Tonry, J. 1983, Ap. J. Suppl., 52, 89.

IRAS Catalogs and Atlases Explanatory Supplement. 1985, ed. C. A. Beichman, G. Neugebauer, H. J. Habing, P. E. Clegg, and T. J. Chester, (Washington, DC: US Government Printing Office).

IRAS Catalogs and Atlases: Point Source Catalog. 1985(Washington, DC: US Government Printing Office)

IRAS Catalogs and Atlases: Small Scale Structure Catalog. 1986, prepared by G. Helou and D. Walker (Washington, DC: US Government Printing Office).

IRAS Catalogs and Atlases: Introduction to the Small Scale Structure Catalog. 1986, ed. G. Helou, (Washington, DC: US Government Printing Office).

Johnson, H. L. 1966, Ann. Rev. Astr. Ap., 4, 193.

Kirschner, R. P., Oemler, A., and Schechter, P. L. 1978, A.J., 83, 1549.

Kleinmann, S., and Keel, W. 1987, in Star Formation in Galaxies, ed. C. J. Persson, (Washington, DC: US Government Printing Office), in press.

Lawrence, A., Walker, D., Rowan-Robinson, M., Leech, K. J., and Penston, M. V. 1986, M.N.R.A.S., 219, 687

Low, F. J., et al. 1984, Ap. J., 278, L19.

Malkan, M. 1983, Ap. J., 268, 582.

Malkan, M., and Sargent, W. L. W. 1982, Ap. J., 25, 22.

Meiksin, A., and Davis, M. 1986, A.J., 91, 191.

Miley, G., Neugebauer, G., and Soifer, B. T. 1985, Ap. J. (Letters), 293, L11.

Neugebauer, G., Soifer, B. T., and Miley, G. 1985, Ap. J. (Letters), 295, L27.

Ney, E. P., et al. 1975, Ap. J.(Letters), 198, L 129.

Nilson, P. 1973, Uppsala General Catalog of Galaxies, Vol. 1, (Uppsala:

Uppsala Offset Center).

O'Dell, S. L., Scott, H. A., and Stein, W. A. 1986, preprint.
Oke, J. B., and Gunn, J. E. 1982, Pub. A.S.P., 94, 586

Palumbo, G. G. C., Tanzella-Nitta, G., and Vettolani, G. 1983, Catalog of Radial Velocities of Galaxies (New York: Gordon \& Breach).

Persson, C. J., and Helou, G. 1986, Ap. J., 314, 513.

Rice, W. L., Lonsdale, C. J., Soifer, B. T., Neugebauer, G., and Kopan, E. L. 1987, Ap.J.Suppl, in preparation.

Rieke, G. H., and Lebofsky, M. 1986, Ap. J., 304, 326.

Rieke, G. H., Lebofsky, M. J., Thompson, R. I., Low, F. J., and Tokunaga, A. T. 1980, Ap.J., 238, 24.

Rowan-Robinson, M., and Crawford, J. 1987, in Star Formation in Galaxies, ed. C. J. Persson (Washington, DC: US Government Printing Office), in press.

Sandage, A. 1975, in Galaxies and the Universe, ed. A. Sandage, M. Sandage, and J. Kristian (Chicago: University of Chicago Press), p. 761.

Sandage, A., and Tammann, G. A. 1981, A Revised Shapely-Ames Catalog of Bright Galaxies (Washington, DC: Carnegie Institute).

Sanders, D. B., Scoville, N. Z., Young, J. S., Soifer, B. T., Schloerb, F. P., Rice, W. L., and Danielson, G. E. 1986, Ap. J., (Letters), 305, L45.

Sanders, D. B., et al. $1987 a$, in preparation.

$1987 \mathrm{~b}$, in preparation.

Schechter, P. 1976, Ap. J., 203, 297.

Schmidt, M. 1968, Ap. J., 151, 393.

Schmidt, M., and Green, R. 1983, Ap. J., 269, 352.

Smith, B. J., Kleinmann, S. G., Huchra, J. P., and Low, F. J. 1987, in Star Formation in Galaxies, ed. C. J. Persson, (Washington, DC: US Government Printing Office), in press.

Soifer, B. T., et al. 1984, Ap. J. (Letters), 278, L71.

Soifer, B. T., Sanders, D. B., Neugebauer, G., Danielson, G. E., Lonsdale, C. J., Madore, B. F., and Persson, S. E. 1986, Ap. J. (Letters), 303, L41 (Paper I).

Thuan, T. X., and Martin, G. E. 1981, Ap. J., 247, 823.

Vader, J. P. 1986, IAU Circ. No. 4265.

Yahil, A., Walker, D., and Rowan-Robinson, M. 1986, Ap. J. (Letters), 301, L1.

Yahil, A., Sandage, A. R., and Tammann, G. A. 1980, Ap. J., 242, 448.

Zwicky, F. et al. 1961-1968, Catalog of Galaxies and Clusters of Galaxies (Pasadena: California Institute of Technology).

G. E. Danielson: Palomar Observatory and Division of Geology and Planetary Science, California Institute of Technology, Mudd 170-25, Pasadena, CA 91125

J. Elias, B. F. Madore, G. Neugebauer, D. B. Sanders, and B. T. Sofier: California Institute of Technology, Downs 320-47, Pasadena, CA 91125

Carol J. Lonsdale, and W. L. Rice: IPAC, Mail Code 100-22, California Institute of Technology, Pasadena, CA 91125 